\title{
MAGNETIC PROPERTIES OF SOLAR ACTIVE REGIONS THAT GOVERN LARGE SOLAR FLARES AND ERUPTIONS
}

\author{
Shin Toriumi $^{1}$, Carolus J. SchriJver ${ }^{2}$, Louise K. Harra ${ }^{3}$, Hugh Hudson ${ }^{4}$, and Kaori Nagashima ${ }^{5}$ \\ ${ }^{1}$ National Astronomical Observatory of Japan, 2-21-1 Osawa, Mitaka, Tokyo 181-8588, Japan; shin.toriumi@nao.ac.jp \\ ${ }^{2}$ Lockheed Martin Advanced Technology Center, 3251 Hanover Street, Palo Alto, CA 94304, USA \\ ${ }^{3}$ UCL-Mullard Space Science Laboratory, Holmbury St Mary, Dorking, Surrey, RH5 6NT, UK \\ ${ }^{4}$ SUPA School of Physics and Astronomy, University of Glasgow, UK \\ ${ }^{5}$ Max-Planck-Institut für Sonnensystemforschung, Justus-von-Liebig-Weg 3, D-37077 Göttingen, Germany \\ Received 2016 August 6; revised 2016 November 14; accepted 2016 November 14; published 2016 December 30
}

\begin{abstract}
Solar flares and coronal mass ejections (CMEs), especially the larger ones, emanate from active regions (ARs). With the aim of understanding the magnetic properties that govern such flares and eruptions, we systematically survey all flare events with Geostationary Orbiting Environmental Satellite levels of $\geqslant$ M5.0 within $45^{\circ}$ from disk center between 2010 May and 2016 April. These criteria lead to a total of 51 flares from 29 ARs, for which we analyze the observational data obtained by the Solar Dynamics Observatory. More than $80 \%$ of the 29 ARs are found to exhibit $\delta$-sunspots, and at least three ARs violate Hale's polarity rule. The flare durations are approximately proportional to the distance between the two flare ribbons, to the total magnetic flux inside the ribbons, and to the ribbon area. From our study, one of the parameters that clearly determine whether a given flare event is CME-eruptive or not is the ribbon area normalized by the sunspot area, which may indicate that the structural relationship between the flaring region and the entire AR controls CME productivity. AR characterization shows that even X-class events do not require $\delta$-sunspots or strong-field, high-gradient polarity inversion lines. An investigation of historical observational data suggests the possibility that the largest solar ARs, with magnetic flux of $2 \times 10^{23} \mathrm{Mx}$, might be able to produce "superflares" with energies of the order of $10^{34} \mathrm{erg}$. The proportionality between the flare durations and magnetic energies is consistent with stellar flare observations, suggesting a common physical background for solar and stellar flares.
\end{abstract}

Key words: Sun: activity - Sun: coronal mass ejections (CMEs) - Sun: flares - Sun: magnetic fields - sunspots

\section{INTRODUCTION}

Solar flares and coronal mass ejections (CMEs) are the most catastrophic energy-conversion phenomena in the present solar system. It is now widely accepted that flares are associated with magnetic reconnection, a physical process that rearranges the magnetic configuration and converts magnetic energy into kinetic energy and thermal energy, and with nonthermal particle acceleration (Priest \& Forbes 2002; Shibata \& Magara 2011). Observations show that similar flaring phenomena are found in a wide variety of stars (Benz \& Güdel 2010).

Since their discovery by Carrington (1859) and Hodgson (1859), solar flares, especially the larger ones, are known to appear in and around active regions (ARs), including sunspots. Observationally, the complex ARs called $\delta$-sunspots, in which umbrae of positive and negative polarities share a common penumbra, tend to produce larger flare eruptions (Künzel 1960; Zirin \& Liggett 1987; Sammis et al. 2000). In the $\delta$-spots, the neighboring polarities are likely to possess a strong-field, highgradient, highly sheared polarity inversion line (PIL), which indicates the existence of intense currents that can store free magnetic energy above in the corona (Schrijver 2007).

As the flare evolves, the two ribbons extend around the sheared PIL, which is observed in $\mathrm{H} \alpha$ and other chromospheric lines (e.g., Dodson 1949; Bruzek 1964; Asai et al. 2004). In the standard model for eruptive flares, the CSHKP model (Carmichael 1964; Sturrock 1966; Hirayama 1974; Kopp \& Pneuman 1976), the flare ribbons are caused by magnetic reconnection through the precipitation of high-energy electrons and the effect of thermal conduction, and thus they indicate the footpoints of newly reconnected field lines (post-flare loops).
CMEs are often associated with flares, particularly with the more energetic ones (e.g., Webb \& Hundhausen 1987; Andrews 2003; Yashiro et al. 2005; Hudson 2010). The studies by, e.g., Wang \& Zhang (2007), Cheng et al. (2011), Kahler et al. (2015), Thalmann et al. (2015), and Sun et al. (2015) suggest a trend that CME-eruptive flares occur at larger distances from the AR centers and have larger "decay index" values (Kliem \& Török 2006) than the non-eruptive ones (also referred to as confined flares or failed eruptions). Although a general picture begins to emerge from these studies, each of them is based on a small sample of selected flares from different regions (some along with nonflaring counterparts) or on a small set of events from one target region. In a recent analysis, Harra et al. (2016) used the set of X-class flares from the current solar cycle to show that there is no obvious difference in the flare duration between the eruptive and noneruptive events and that the non-eruptive ones tend to have a larger spot area. In this study, we expand the sample used by Harra et al. (2016) by lowering the threshold to mid-M-class flares and by expanding the time interval (see Section 2.1). This also enables us to test the findings from the studies referenced earlier in this paragraph using a much larger sample in which the only selection bias is that they do not occur too far from disk center, so that their magnetic patterns are well observed.

One of the ultimate goals of this paper is to find the physical parameters that dictate the peak magnitudes and timescales of the Geostationary Orbiting Environmental Satellites (GOES) soft X-ray (SXR) flux (GOES parameters) and that determine whether a given flare becomes CME-eruptive or not, and in case of eruption, the speed of the CME (CME parameters). For this purpose, we carry out a systematic survey of observational 
data of flaring ARs obtained by the Solar Dynamics Observatory (SDO; Pesnell et al. 2012). We especially evaluate the morphological properties of such flaring ARs for characterizing the magnetic environment of flare eruptions (AR parameters), as well as those of the flare ribbons as indicators of magnetic fields in flare reconnection (flare parameters), and investigate the statistical relationships among the GOES parameters, CME parameters, AR parameters, and flare parameters.

Another aim is to elucidate the formation process of flaring ARs. Since such large-scale ARs are created by flux emergence, the transportation of dynamo-generated magnetic flux in the deeper interior to the solar surface (Parker 1955), it is of necessity to conduct numerical simulations and model the flux emergence (Fan 2009; Cheung \& Isobe 2014), which is, however, beyond the scope of this paper. Instead, we here characterize the flaring ARs by focusing on the positional relationship between the flare-related ribbons (closely associated with the PIL) and the entire AR and examine the statistical tendencies.

To understand the diversity of flare events, such as extreme solar events in history, simply surveying the SDO data set may not be sufficient. One possible way to fulfill this desire is to learn from the past. Therefore, as an example, we also introduce a great flare event that occurred in 1946 (SOL1946-07-25) and explore the possibilities of historical data analysis in combination with our statistical results.

The rest of the paper is organized as follows. In Sections 2 and 3, we describe the data analysis and show the statistical results, respectively. Then, Section 4 is dedicated to characterizing the flaring ARs and their statistical trends, while in Section 5 we show the analysis on our historical flare event. We discuss the results in Section 6, and in Section 7 we conclude the paper.

\section{DATA ANALYSIS}

\subsection{Event Selection and Parameters}

For the purpose of this study, we analyzed all flare events with GOES SXR magnitudes greater than or equal to M5.0 within $45^{\circ}$ from the disk center in the period from 2010 May to 2016 April, namely, $6 \mathrm{yr}$ from the beginning to the declining phase of solar cycle 24 . These constraints led us to a total of 51 flares from 29 regions with distinct NOAA AR numbers. Table 1 summarizes the target 51 flare events. For each event, we obtained these GOES parameters from the GOES SXR (1-8 A channel) light curve:

1. FWHM of the light curve, $\tau_{\text {FWHM; }}$

2. $e$-folding decay time, $\tau_{\text {decay }}$; and

3. peak flux, $F_{\mathrm{SXR}}$.

We give the method of estimation of the two timescales in Section 2.2.

In order to identify the physical parameters that may characterize the flare events, we used the observational data taken by the Helioseismic and Magnetic Imager (HMI; Scherrer et al. 2012; Schou et al. 2012) and the Atmospheric Imaging Assembly (AIA; Lemen et al. 2012) aboard SDO. For each event, we made projection-corrected tracked data cubes of the intensitygram, line-of-sight (LOS) magnetogram, and $1600 \AA$ images, using the mtrack module (Bogart et al. 2011). From the intensitygram and $1600 \AA$ data, center-to-limb variations were subtracted based on the method introduced in Toriumi et al. (2014a). The data cubes have a cadence of $180 \mathrm{~s}$ for magnetogram and intensitygram and $120 \mathrm{~s}$ for $1600 \AA$ images, both having pixel size 1 !' 0 . The sequence of each data cube is 2 or $3 \mathrm{hr}$ starting from before the GOES start time (typically 20 minutes before), while the area of the field of view (FOV) is $512^{\prime \prime} \times 256^{\prime \prime}$ or $1024^{\prime \prime} \times 512^{\prime \prime}$ centering the target AR.

From the first frames of the intensitygram and magnetogram of each flare event, which is well before the flare peak (typically 40 minutes before), we selected the following AR parameters:

1. sunspot area, $S_{\text {spot }}$, which is the total area of umbrae and penumbrae of an AR, defined in this study as the deprojected area of the pixels with intensity less than $85 \%$ of the mean quiet-Sun intensity;

2. total unsigned flux, $|\Phi|_{\mathrm{AR}}=\int_{S_{\mathrm{FOV}}}|B| d s$, where $S_{\mathrm{FOV}}$ $\left(=512^{\prime \prime} \times 256^{\prime \prime}\right.$ or $\left.1024^{\prime \prime} \times 512^{\prime \prime}\right)$ is the FOV area, ${ }^{6} B$ is the LOS magnetic flux density in each pixel, and $s$ $\left(=1^{\prime \prime} \times 1^{\prime \prime}\right)$ is the pixel area; and

3. normalized field strength, which is the total AR flux normalized by the spot area, $|\bar{B}|_{\mathrm{AR}}=|\Phi|_{\mathrm{AR}} / S_{\mathrm{spot}}$.

Here, the spot areas were measured in millionths of the solar hemisphere (MSH), which is equivalent to $3.0 \times 10^{6} \mathrm{~km}^{2}$.

For each event, we extracted the flare ribbons from $1600 \AA$ images by defining them as the pixels with intensity at any time during the flare (until the last frame) equal to or larger than $40 \sigma$ (standard deviation) above the mean of the quiet-Sun values, and we made binary ribbon maps. After removing the saturated frames, we stacked the binary ribbon maps over time and made a binary ribbon composite. By plotting the ribbon composite over the magnetogram (first frame), we divide the composite into two parts, the ribbon in the positive polarity and that in the negative polarity. Then, the flare parameters were defined as follows:

1. ribbon area, $S_{\text {ribbon }}$, the total area of the ribbon composite;

2. ribbon distance, $d_{\text {ribbon }}$, the separation between the two area-weighted (i.e., geometrical) centroids of the ribbons in the positive and negative polarities;

3. total unsigned flux inside the ribbon, $|\Phi|_{\text {ribbon }}=$ $\int_{S_{\text {ribbon }}}|B| d s ;$ and

4. normalized field strength of the ribbon, $|\bar{B}|_{\text {ribbon }}=$ $|\Phi|_{\text {ribbon }} / S_{\text {ribbon }}$.

In addition, we determined whether each flare event was CME-eruptive or not (i.e., confined) by reference to the CME catalog ${ }^{7}$ of the Solar and Heliospheric Observatory $(\mathrm{SOHO}) /$ Large Angle Spectroscopic Coronagraph. For eruptive events,

1. CME speed, $V_{\mathrm{CME}}$, the linear speed obtained by fitting a straight line to the height-time measurements,

was also listed from the catalog as a CME parameter. Note that because the current analysis is based only on the flare events within $45^{\circ}$ from the disk center, there is a potential to miss

\footnotetext{
6 Depending on the target AR, we used an FOV of $512^{\prime \prime} \times 1024^{\prime \prime}$ or $1024^{\prime \prime} \times 2048^{\prime \prime}$ to cover it. However, in some cases, the rectangular FOV contains the neighboring flux concentrations that may not be related to the target region. We masked such flux concentrations to obtain a better AR area (see Figure 12).

7 http://cdaw.gsfc.nasa.gov/CME_list/
} 
Table 1

Properties of Flare Events

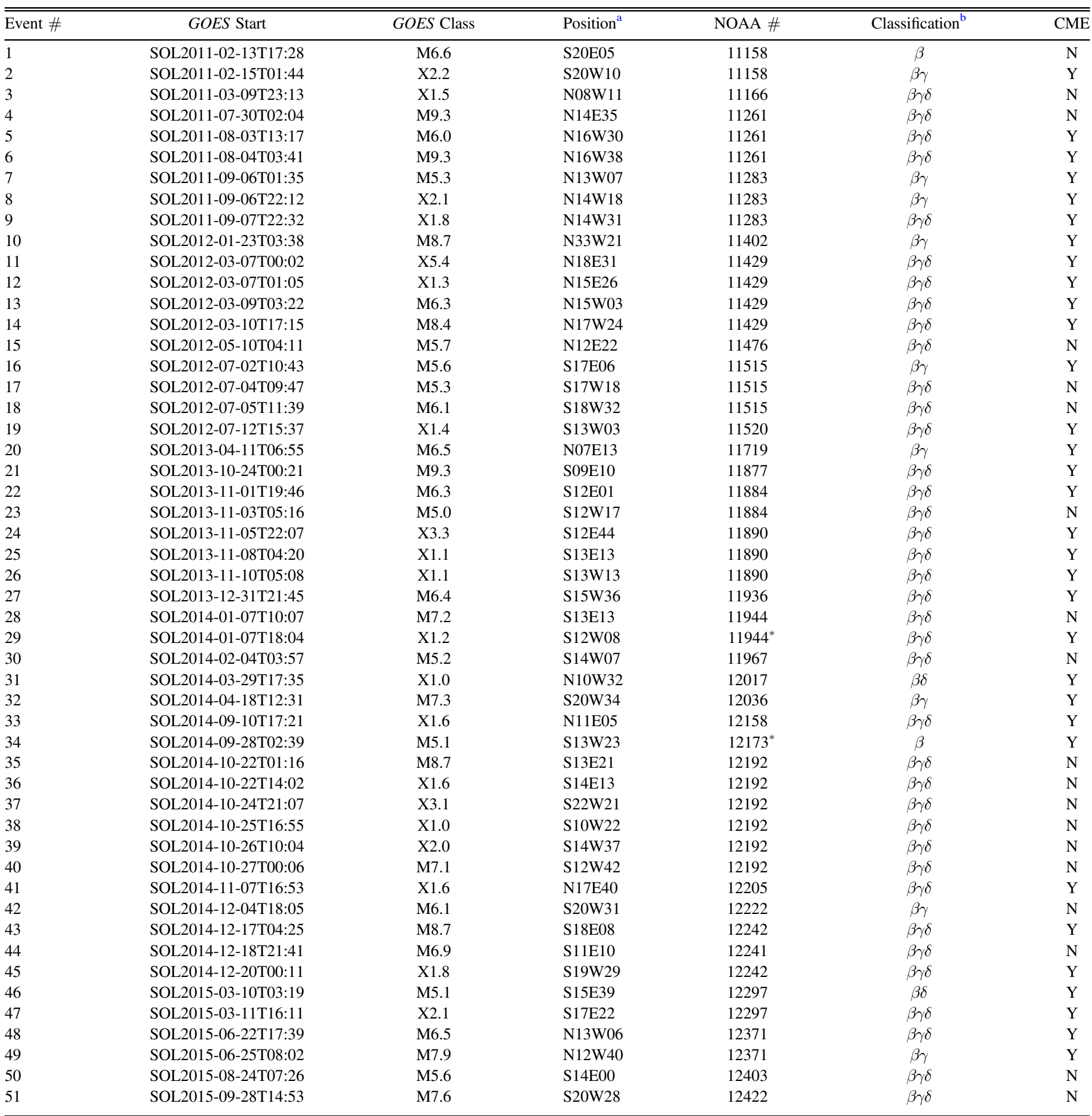

Notes. NOAA number with asterisk $(*)$ indicates inter-AR flare. Event \#29 occurred between NOAA ARs 11944 and 11943 , and \#34 between ARs 12173 and 12172.

${ }^{a}$ Heliographic position.

${ }^{\mathrm{b}}$ Mount Wilson sunspot classification on the day of the flare occurrence provided by NOAA/USAF. $\beta$ is assigned to a sunspot group that has both positive and negative polarities. $\gamma$ indicates that a sunspot group has a complex region of multiple spots with intermixed polarity. $\delta$ indicates that at least one sunspot contains opposite polarities inside a common penumbra separated by no more than $2^{\circ}$ in heliographic distance.

some CMEs: Yashiro et al. (2005) suggest that roughly one in six CMEs are missed from the on-disk M-class events (see Table 3 of their paper).
Furthermore, we followed the long-term evolution of each AR by making HMI data cubes that cover the AR's whole disk passage. 
2014-10-24 X3.1-class flare NOAA AR 12192
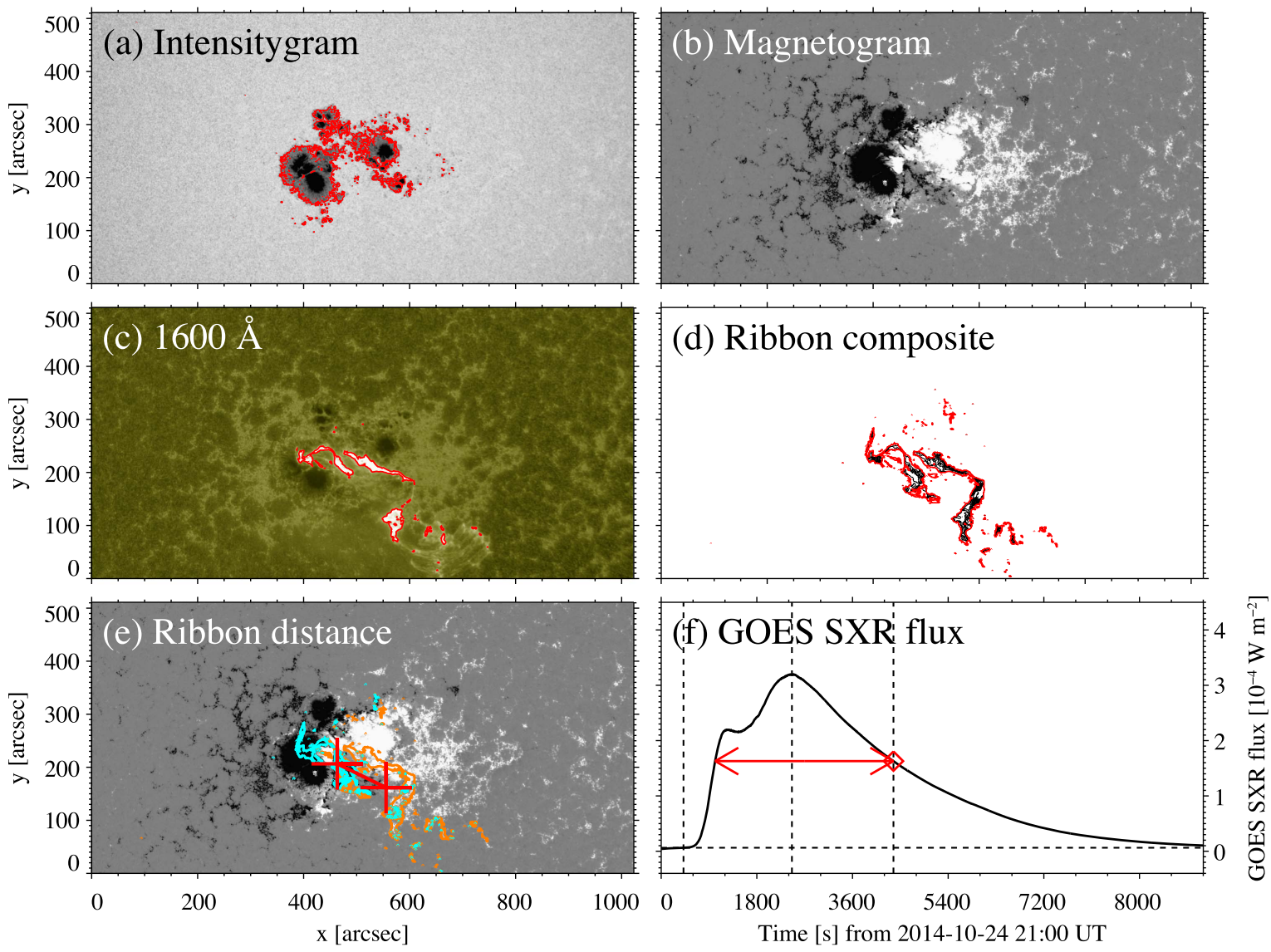

Figure 1. Sample flare data: X3.1-class event in NOAA AR 12192 (event \#37). (a) HMI intensitygram and (b) magnetogram (saturating at $\pm 400 \mathrm{G}$ ), both taken at 2014-10-24 21:00 UT. The red contour in panel (a) surrounds the umbrae and penumbrae, defining the spot area. (c) AIA 1600 $\AA$ image at 21:20 UT, with red contour defining the flare ribbon (intensity of $\geqslant 40 \sigma$ above the mean) in this frame. (d) Detected flare ribbons from some selected frames are overlaid (black). The red contour outlining these ribbons indicates the ribbon composite. (e) Composite ribbons in the positive (orange) and negative (turquoise) polarities plotted over the magnetogram (b). Red plus signs show the area-weighted centroids of the two ribbons. A red straight line connects the two centroids, indicating the ribbon distance. (f) GOES SXR 1-8 Å flux (solid curve). Three vertical dashed lines show (from left to right) the GOES start (21:07 UT), peak (21:47 UT), and end (22:13 UT) times. The horizontal dashed line indicates the background level, which is the flux measured at the GOES start time. After subtracting this background level from the light curve, FWHM time is measured (red arrow), while the flux and its time derivative at the GOES end time (red diamond) are used for measuring the $e$-folding decay time.

\subsection{Sample Event}

Figure 1 shows an example of the analyzed data sets: the X3.1-class event in NOAA AR 12192. From the first frames of the HMI intensitygram and magnetogram (panels (a) and (b)), we measured the spot area $S_{\text {spot }}$, total flux $|\Phi|_{\mathrm{AR}}$, and normalized field strength $\left.\overline{\mid B}\right|_{\text {AR }}$. By temporally stacking the flare ribbons extracted from the AIA $1600 \AA$ images (panel (c)), we made a ribbon composite (panel (d)), which provides our measure of the ribbon area $S_{\text {ribbon. }}$ Then, by overlaying the ribbon composite on the magnetogram (panel (e)), we measured the ribbon distance $d_{\text {ribbon }}$, the total flux $|\Phi|_{\text {ribbon }}$, and the mean field strength $|\bar{B}|_{\text {ribbon. }}$. Panel (f) shows the GOES SXR (1-8 $\AA$ ) light curve. For measuring the FWHM time $\tau_{\text {FWHM }}$, the background level, which is the flux at the GOES start time, is first subtracted from the light curve. The $e$-folding decay time $\tau_{\text {decay }}$ is calculated using the flux $F_{\text {SXR }}(t)$ and its time derivative $d F_{\text {SXR }}(t) / d t$ at the GOES end time as $\tau_{\text {decay }}=-F_{\text {SXR }}(t) /\left(d F_{\text {SXR }}(t) / d t\right)$.

Since the flare ribbons expand as the flare evolves (Section 1), we need to take into account the effect of this expansion, especially the timing when the evolution slows. Figure 2 compares the GOES light curve, the evolution of the ribbon composite area $S_{\text {ribbon }}(t)$ (ribbon composite made from the AIA $1600 \AA$ data sets until each moment $t$ ), and the evolution of the ribbon distance $d_{\text {ribbon }}(t)$ (ribbon distance measured from $\left.S_{\text {ribbon }}(t)\right)$. The final values of these parameters are used in the analysis as $S_{\text {ribbon }}$ and $d_{\text {ribbon. }}$. In the middle panel, we measure the actual ribbon area at each moment $t$ and overplot it as $S_{\text {ribbon }}^{*}(t)$.

Although the most impulsive period is not seen due to saturation in the $1600 \AA$ images, $S_{\text {ribbon }}^{*}(t)$ reaches its maximum in the rising phase before the GOES peak time. This is reasonable because the ribbons indicate the heating of chromospheric plasma via thermal conduction and high-energy electrons driven by the reconnection, while the SXR loops are formed following chromospheric evaporation (see, e.g., Shibata \& Magara 2011). This may be one manifestation of the socalled Neupert effect (Neupert 1968). As a result, images showing the evolutions of $S_{\text {ribbon }}(t)$ and thus $d_{\text {ribbon }}(t)$ become saturated around the GOES peak.

In this study, the end times of the $S D O$ data sets are well after the GOES peak times, and thus we can consider that the ribbon composite of each flare event sufficiently reflects the expansion of bright ribbons. 


\section{Temporal evolution}
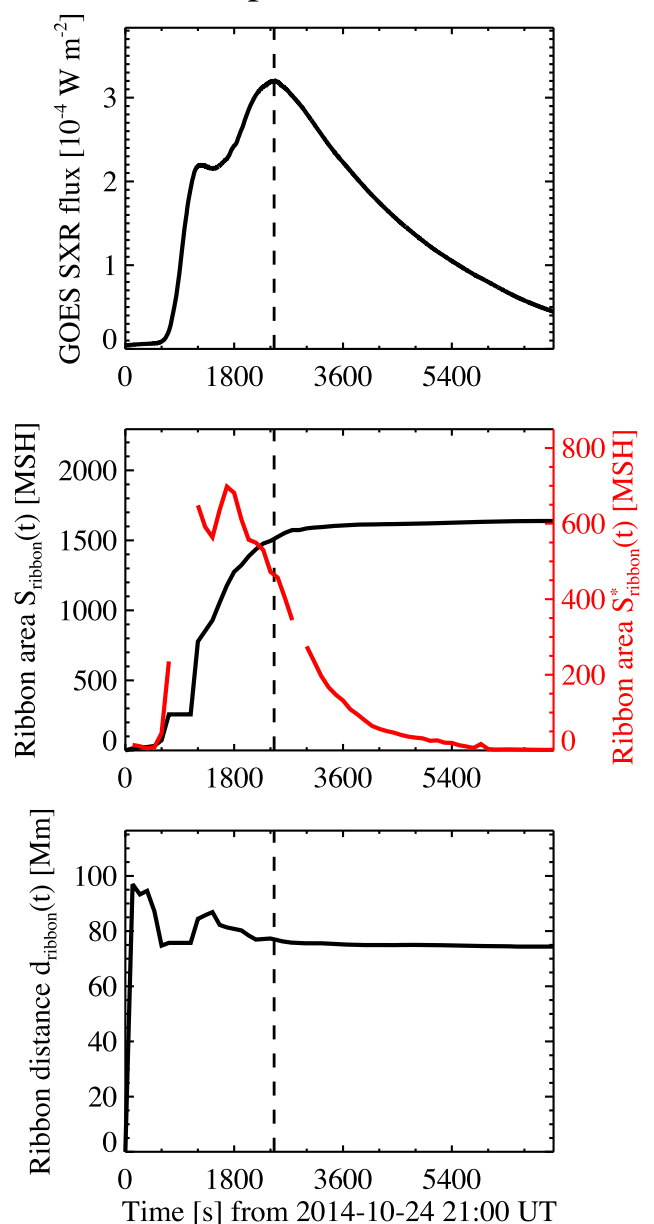

Figure 2. Temporal evolution of the X3.1-class flare (event \#37). Top: GOES SXR 1-8 A flux. Middle: area of the ribbon composite, $S_{\text {ribbon }}(t)$ (black), which is calculated from the AIA $1600 \AA$ images until each moment, $t$, and the actual area of the ribbon at each moment, $S_{\text {ribbon }}^{*}(t)$ (red). The periods of blank $S_{\text {ribbon }}^{*}(t)$ indicate the saturation in the $1600 \mathrm{~A}$ images. Bottom: ribbon distance, $d_{\text {ribbon }}(t)$, which is calculated from the ribbon composite at each moment $S_{\text {ribbon }}(t)$. In all panels, the GOES peak time is indicated with a vertical dashed line, which separates the impulsive phase and the gradual (decay) phase.

\section{STATISTICAL RESULTS}

\subsection{Properties of ARs and Flare Events}

Table 1 shows the Mount Wilson sunspot classification. Here, 23 out of the 29 ARs (79\%) show a $\delta$-structure at least for one flare occurrence. However, although AR 11158 was reported as non- $\delta$, this region actually shows a $\delta$-configuration when it produces the flares. Therefore, the actual fraction increases to $83 \%$ for the $\geqslant$ M5 events under study. This result is in line with previous results that the $\delta$-spots have higher flare productivity (e.g., Sammis et al. 2000).

There are three ARs (10\%) that violate Hale's polarity rule for at least one flare (ARs 11429, 11719, and 12158). If we also count AR 12242, which shows anti-Hale structure until about 1 day before the flare eruption, this fraction becomes $14 \%$. Although this number is much larger than the typically reported value of 3\%-5\% for all ARs (Richardson 1948; Wang \& Sheeley 1989; Khlystova \& Sokoloff 2009), the small sample number does not allow any firm conclusion about this.

The analyzed 51 flares are composed of $20 \mathrm{X}$ - and 31 M-class events, ranging from M5.0 to X5.4. They include several major flares from well-studied ARs. Among others, NOAA AR 11158 produced the first X-class (X2.2) flare in solar cycle 24 (e.g., Schrijver et al. 2011), AR 11429 produced the largest (X5.4) flare so far in this cycle (e.g., Wang et al. 2014), AR 12017 produced the "best-observed" X1.0class flare (e.g., Kleint et al. 2015), and AR 12192, the largest sunspot group so far in the cycle, produced many (6 X- and 24 M-class) but CME-poor events (e.g., Sun et al. 2015; four Xand two M-class events are listed in Table 1). Almost all the events in this table occurred at PILs within the AR's magnetic structure itself. However, there are two exceptional cases: events \#29 (X1.2) and \#34 (M5.1) occurred at the PIL between two neighboring ARs.

\subsection{Parameters That Dictate GOES Light Curves}

In this study, from the $S D O$ data set of each flare event, we measured various parameters: GOES parameters (durations $\tau_{\text {FWHM }}$ and $\tau_{\text {decay }}$ and GOES flux $F_{\text {SXR }}$ ), AR parameters (spot area $S_{\text {spot }}$, total flux $|\Phi|_{\mathrm{AR}}$, and field strength $\left.|\bar{B}|_{\mathrm{AR}}\right)$, and flare parameters (ribbon area $S_{\text {ribbon }}$, distance $d_{\text {ribbon }}$, total flux $|\Phi|_{\text {ribbon }}$, and field strength $\left.|\bar{B}|_{\text {ribbon }}\right)$. The values for all events and their maximum, minimum, median, and standard deviation values are shown in Table 3 of Appendix A. But here we list the ranges and medians of these parameters: $\tau_{\mathrm{FWHM}}=154-4790 \mathrm{~s}$ (median: $1198 \mathrm{~s}), \quad \tau_{\text {decay }}=32-1986 \mathrm{~s} \quad(433 \mathrm{~s}), \quad F_{\text {SXR }}=(0.5-5.4) \times$ $10^{-4} \mathrm{~W} \mathrm{~m}^{-2}\left(0.87 \times 10^{-4} \mathrm{~W} \mathrm{~m}^{-2}\right), \quad S_{\text {spot }}=126-2877 \mathrm{MSH}$ (781 MSH), $|\Phi|_{\mathrm{AR}}=(1.1-16.6) \times 10^{22^{2}} \mathrm{Mx}\left(3.8 \times 10^{22} \mathrm{Mx}\right)$, $|B|_{\mathrm{AR}}=568-810 \mathrm{G}(685 \mathrm{G}), S_{\text {ribbon }}=102-1639 \mathrm{MSH}(431$ $\mathrm{MSH}), \quad d_{\text {ribbon }}=4.1-105.1 \mathrm{Mm} \quad(26.9 \mathrm{Mm}), \quad|\Phi|_{\text {ribbon }}=$ $(0.9-16.1) \times 10^{21} \quad \mathrm{Mx} \quad\left(4.4 \times 10^{21} \mathrm{Mx}\right), \quad$ and $\mid \bar{B}_{\text {ribbon }}=$ $125-590 \mathrm{G}(308 \mathrm{G})$.

In order to find the physical parameters that dictate the GOES light curves, we made scatter plots of the measured data, namely, the scatter plots of $y=\left\{\tau_{\mathrm{FWHM}}, \tau_{\text {decay }}, F_{\mathrm{SXR}}\right\}$ versus $x=\left\{S_{\text {spot }},|\Phi|_{\mathrm{AR}},|\bar{B}|_{\mathrm{AR}}, S_{\text {ribbon }}, d_{\text {ribbon }},|\Phi|_{\text {ribbon }},|\bar{B}|_{\text {ribbon }}\right\}$. For $x$, we also used the ratio of the two areas, $S_{\text {ribbon }} / S_{\text {spot }}$, ranging from $9.0 \%$ to $328 \%$ (median: $56 \%$ ), and that of total fluxes, $|\Phi|_{\text {ribbon }} /|\Phi|_{\text {AR }}=1.6 \%-43 \%$ (11\%). Then, for obtaining the empirical relationship, we evaluated for each diagram the power-law index $\alpha$ by fitting the data with a power-law function, $\log y=\alpha \log x+$ const., or $y \propto x^{\alpha}$. We also measured the correlation coefficient, $\mathrm{CC}(\log x, \log y)$, to estimate the degree of dispersion of each plot. Note that we assumed errors for the $y$-coordinate only.

As a result, we obtained 27 scatter plots and thus 27 empirical relations, whose power-law indices $\alpha$ and correlation coefficients CC are summarized in Table 2. Figure 3 displays the six least-scattered plots (strongest correlations with $|C C| \geqslant 0.6$, shown in bold in Table 2).

The best correlations are obtained from the scatter plots of the FWHM duration of the flares, $\tau_{\text {FWHM }}$ (Figures 3(a)-(c)). They are of the flare parameters: the ribbon distance, $d_{\text {ribbon }}$,

$$
\log \tau_{\text {FWHM }}=(0.96 \pm 0.09) \log d_{\text {ribbon }}+(1.67 \pm 0.13),
$$

the ribbon total flux, $|\Phi|_{\text {ribbon }}$,

$$
\log \tau_{\text {FWHM }}=(1.04 \pm 0.12) \log |\Phi|_{\text {ribbon }}+(-19.4 \pm 2.51),
$$

and the ribbon area, $S_{\text {ribbon, }}$,

$$
\log \tau_{\mathrm{FWHM}}=(1.10 \pm 0.15) \log S_{\text {ribbon }}+(0.08 \pm 0.40),
$$


Table 2

Summary of Power-law Indices and Correlation Coefficients

\begin{tabular}{|c|c|c|c|c|c|c|c|c|}
\hline \multirow{2}{*}{ Parameter } & \multicolumn{2}{|c|}{$\tau_{\text {FWHM }}$} & \multicolumn{2}{|c|}{$\tau_{\text {decay }}$} & \multicolumn{2}{|c|}{$F_{\text {SXR }}$} & \multicolumn{2}{|c|}{$V_{\mathrm{CME}}$} \\
\hline & $\alpha$ & $\mathrm{CC}$ & $\alpha$ & $\mathrm{CC}$ & $\alpha$ & $\mathrm{CC}$ & $\alpha$ & $\mathrm{CC}$ \\
\hline$S_{\text {spot }}$ & $0.43 \pm 0.17$ & 0.35 & $0.25 \pm 0.19$ & 0.18 & $0.17 \pm 0.25$ & 0.25 & $0.34 \pm 0.17$ & 0.34 \\
\hline$\left.\overline{\mid B}\right|_{\mathrm{AR}}$ & $-0.48 \pm 1.62$ & -0.04 & $0.30 \pm 0.23$ & 0.18 & $-2.29 \pm 0.85$ & -0.36 & $-2.72 \pm 1.20$ & -0.38 \\
\hline$S_{\text {ribbon }}$ & $1.10 \pm \mathbf{0 . 1 5}$ & 0.72 & $\mathbf{1 . 0 5} \pm \mathbf{0 . 1 8}$ & 0.64 & $0.19 \pm 0.12$ & 0.23 & $0.50 \pm 0.16$ & 0.50 \\
\hline$d_{\text {ribbon }}$ & $\mathbf{0 . 9 6} \pm \mathbf{0 . 0 9}$ & 0.83 & $\mathbf{0 . 8 8} \pm \mathbf{0 . 1 2}$ & 0.71 & $0.13 \pm 0.09$ & 0.20 & $0.38 \pm 0.13$ & 0.47 \\
\hline$S_{\text {ribbon }} / S_{\text {spot }}$ & $0.30 \pm 0.17$ & 0.24 & $0.45 \pm 0.18$ & 0.34 & $-0.04 \pm 0.10$ & -0.06 & $0.12 \pm 0.16$ & 0.14 \\
\hline$|\Phi|_{\text {ribbon }} /|\Phi|_{\mathrm{AR}}$ & $0.79 \pm 0.17$ & 0.54 & $0.91 \pm 0.18$ & 0.58 & $0.14 \pm 0.12$ & 0.17 & $0.36 \pm 0.17$ & 0.37 \\
\hline$\tau_{\text {FWHM }}$ & $\ldots$ & $\ldots$ & $\ldots$ & $\cdots$ & $\ldots$ & $\ldots$ & $0.32 \pm 0.12$ & 0.45 \\
\hline$\tau_{\text {decay }}$ & $\ldots$ & $\cdots$ & $\cdots$ & $\cdots$ & $\ldots$ & $\ldots$ & $0.32 \pm 0.10$ & 0.50 \\
\hline$F_{\mathrm{SXR}}$ & $\ldots$ & $\ldots$ & $\ldots$ & $\ldots$ & $\ldots$ & $\ldots$ & $0.26 \pm 0.20$ & 0.23 \\
\hline
\end{tabular}

Note. Power-law indices $\alpha$ and correlation coefficients CC obtained from various scatter plots are shown. Values with higher correlations $(|\mathrm{CC}| \geqslant 0.6)$ are shown in bold. The quantities in the middle part ( $S_{\text {ribbon }} / S_{\text {spot }}$ and $|\Phi|_{\text {ribbon }} /|\Phi|_{\mathrm{AR}}$ ) are the dimensionless (ratio) parameters, which are the combinations of the parameters in the top part. For $V_{\mathrm{CME}}$ the values for the $\tau_{\mathrm{FWHM}}, \tau_{\text {decay }}$, and $F_{\mathrm{SXR}}$ are also shown in the bottom part.

with correlation coefficients of $\mathrm{CC}=0.83,0.79$, and 0.72 , respectively. Interestingly, all the above equations show powerlaw indices of approximately unity, $\alpha \sim 1$. The other parameters show a more scattered distribution: except for the AR field strength, $|\bar{B}|_{\mathrm{AR}}$, they have positive relations (see Table 2).

The other three best relations are of the $e$-folding decay time, $\tau_{\text {decay }}$ (Figures 3(d)-(f)), and they are of the same flare parameters: the ribbon distance, $d_{\text {ribbon }}$,

$$
\log \tau_{\text {decay }}=(0.88 \pm 0.12) \log d_{\text {ribbon }}+(1.35 \pm 0.18),
$$

the ribbon total flux, $|\Phi|_{\text {ribbon }}$,

$$
\log \tau_{\text {decay }}=(0.96 \pm 0.15) \log |\Phi|_{\text {ribbon }}+(-18.2 \pm 3.21),
$$

and the ribbon area, $S_{\text {ribbon, }}$,

$$
\log \tau_{\text {decay }}=(1.05 \pm 0.18) \log S_{\text {ribbon }}+(-0.21 \pm 0.47),
$$

with $\mathrm{CC}=0.71,0.68$, and 0.64 , respectively. It is natural that $\tau_{\text {decay }}$ also shows strong correlations with the above three parameters, because $\tau_{\text {FWHM }}$ and $\tau_{\text {decay }}$ are highly correlated with each other $(\mathrm{CC}=0.87)$. Although the distributions for $\tau_{\text {decay }}$ are a bit more scattered and thus the correlations are slightly weaker than those of $\tau_{\text {FWHM }}$, the power-law indices still show $\alpha \sim 1$. The other parameters also show similar trends to those of $\tau_{\mathrm{FWHM}}$ with similar power-law indices. However, again, the correlations are on average weaker than those of $\tau_{\text {FWHM }}$.

On the other hand, no diagrams of the GOES peak flux, $F_{\text {SXR }}$, have higher $(|C C| \geqslant 0.6)$ correlations. The maximum correlation coefficient here is just $\mathrm{CC}=0.37$ of the ribbon total flux, $|\Phi|_{\text {ribbon }}$. They show generally positive correlations, but $\left.\overline{\mid B}\right|_{\mathrm{AR}}$ and $S_{\text {ribbon }} / S_{\text {spot }}$ show negative relations.

In this data set, we only have a range of one order of magnitude for the GOES peak flux, $F_{\mathrm{SXR}}=(0.5-5.4) \times 10^{-4} \mathrm{~W} \mathrm{~m}^{-2}$, while the GOES durations span more than one order, $\tau_{\text {FWHM }}=$ $154-4790 \mathrm{~s}$ and $\tau_{\text {decay }}=32-1986 \mathrm{~s}$. This narrow range of $F_{\mathrm{SXR}}$ may be one of the factors that cause the weaker correlations.

\subsection{Parameters That Determine CME Properties}

In our data set of $51 \geqslant$ M5-class events, there are $32 \mathrm{CME}$ eruptive and 19 non-eruptive events. In this section, we search the parameters that determine CME rich/poor and their speed.

Figure 4 displays the histograms for CME-eruptive and noneruptive events. From the top row, one may see that there is no significant difference in distributions of durations and magnitudes between the eruptive and non-eruptive cases. The averages of the log values (indicated by vertical dashed lines) for the eruptive and non-eruptive cases are $\tau_{\mathrm{FWHM}}=1068$ and $826 \mathrm{~s}$ (difference $\left.=26 \%^{8}\right), \tau_{\text {decay }}=386$ and $314 \mathrm{~s}(20 \%)$, and $F_{\text {SXR }}=1.1 \times 10^{-4}$ and $0.83 \times 10^{-4} \mathrm{~W} \mathrm{~m}^{-2}(23 \%)$, respectively. Thus, at least for the $\geqslant$ M5-class events, the longerduration or larger-magnitude flares are not necessarily CMEeruptive.

One of the clear differences is seen in the spot area, $S_{\text {spot }}$. In the middle row of Figure 4, distributions of $S_{\text {spot }}$ show a large discrepancy. Here, the non-eruptive events have larger spot areas. The log averages are $526 \mathrm{MSH}$ for eruptive and 1171 MSH for non-eruptive (difference $=76 \%$ ), and the spot areas of the eruptive cases are significantly smaller than those of the non-eruptive case at the $99.5 \%$ confidence level (see Appendix B). As one might expect, the latter value is to some extent influenced by the six non-eruptive events from the cycle's largest spot group, AR 12192 (Figure 1). However, even without these flares, the log-mean spot area of the remaining 13 events is still $801 \mathrm{MSH}$ (difference $=41 \%$ ) and the distribution difference is significant at $95 \%$ confidence. On the other hand, the distributions and thus the mean values of the ribbon area, $S_{\text {ribbon, }}$ are similar for the eruptive and noneruptive cases: the log means are 432 and $419 \mathrm{MSH}$, respectively (difference $=3.0 \%$ ).

As a result of the differences in $S_{\text {spot }}$ and $S_{\text {ribbon }}$, the area ratios, $S_{\text {ribbon }} / S_{\text {spot }}$, also show a difference in the distributions with $\log$-mean values of 0.82 and 0.36 for the eruptive and non-eruptive cases, respectively (difference $=79 \%$ ). The threshold dividing the two regimes is about 0.5. This clear

\footnotetext{
$\overline{8}$ Hereafter we use relative difference $|a-b| /(|a+b| / 2)$ to show the quantitative difference between $a$ and $b$.
} 


\section{Scatter plots with strongest correlations}
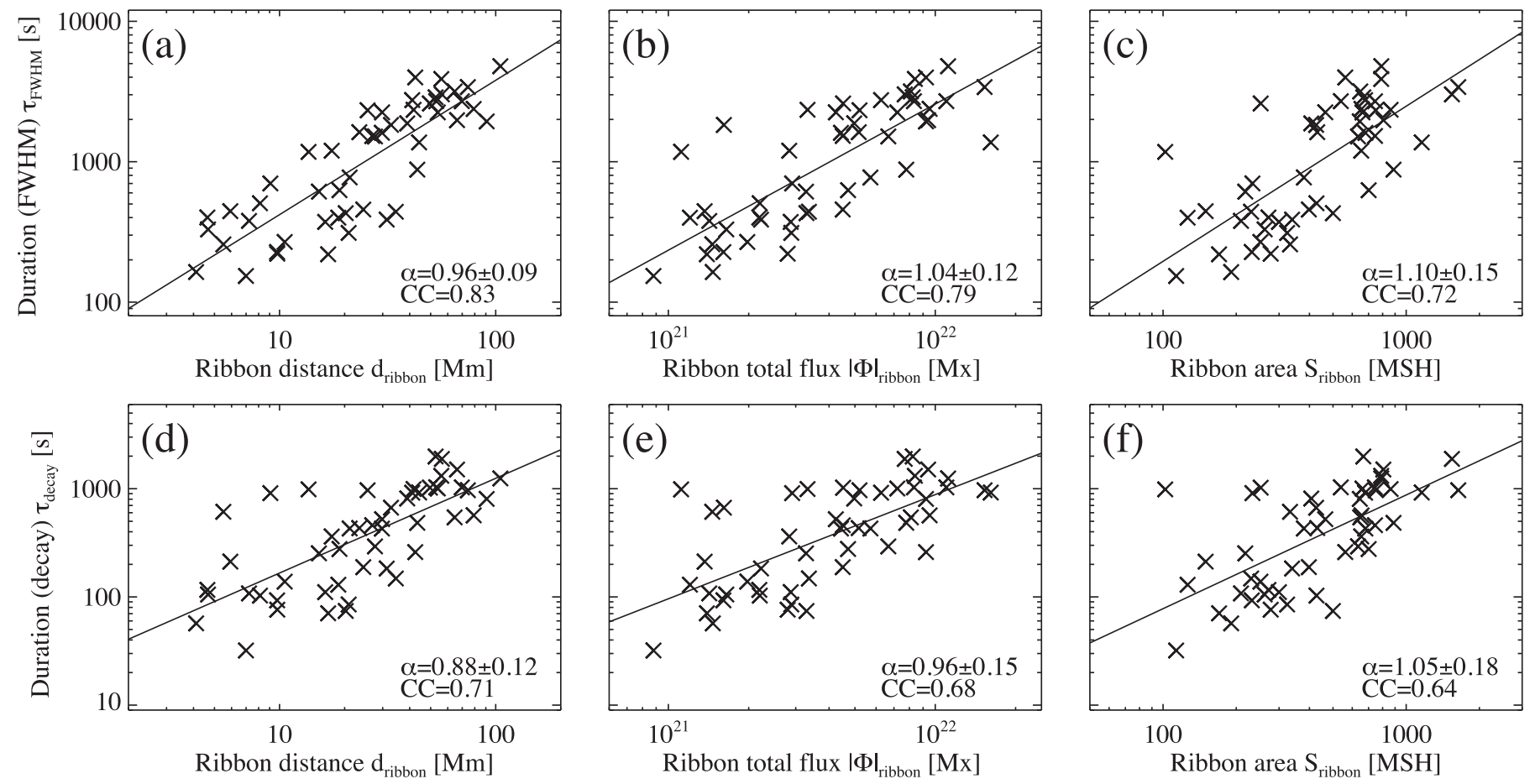

Figure 3. Scatter plots with strongest correlations $(|\mathrm{CC}| \geqslant 0.6)$. In each panel, a straight line shows the result of a linear fitting to the log-log plots, while power-law index $\alpha$ and correlation coefficient CC are shown at the bottom right.

difference may indicate that what determines the CME productivity is the relative structural relation between the magnetic fields of the flaring region (sheared PIL, flare ribbons, flare arcades, etc.) and those of the entire AR.

Tendencies similar to those of the areas $\left(S_{\text {spot }}\right.$ and $\left.S_{\text {ribbon }}\right)$ are seen for the total magnetic flux (bottom row of Figure 4 ). The log-mean values of $|\Phi|_{\mathrm{AR}}$ for the eruptive and non-eruptive cases are $3.2 \times 10^{22}$ and $6.0 \times 10^{22} \mathrm{Mx}$ (difference $=59 \%$; significant at $99.5 \%$ confidence), respectively, while those of $|\Phi|_{\text {ribbon }}$ are $3.8 \times 10^{21}$ and $4.1 \times 10^{21} \mathrm{Mx}(6.5 \%)$, respectively. And thus the log means of $|\Phi|_{\text {ribbon }} /|\Phi|_{\mathrm{AR}}$ are 0.12 and $0.07(53 \%)$, respectively.

The other three parameters, $|B|_{\mathrm{AR}},\left|B_{\text {ribbon }}\right|$, and $d_{\text {ribbon }}$, are not very different between the two cases: the differences are $4.8 \%$, $9.5 \%$, and $7.7 \%$, respectively.

Previous findings of our earlier report (Harra et al. 2016) and of event studies introduced in Section 1 are confirmed by the present comprehensive survey: the present work covers all ondisk flare events over a $6 \mathrm{yr}$ period, including the cycle maximum without selection bias, and extends the on-disk sample of Harra et al. (2016), with the GOES peak brightness reaching down to the M5 level, which is one virtue of this study. ${ }^{9}$

In order to find the parameters that control the CME speed, we made scatter plots of $V_{\mathrm{CME}}$, similar to those in Section 3.2, but this time also of GOES parameters, $\tau_{\mathrm{FWHM}}, \tau_{\text {decay }}$, and $F_{\text {SXR }}$. The rightmost column of Table 2 summarizes the powerlaw indices, $\alpha$, and their correlation coefficients, CC. The largest value is $\mathrm{CC}=0.5$ for the ribbon area $S_{\text {ribbon }}$, ribbon flux

\footnotetext{
9 For example, 20 on-disk flares from all 42 X-class events were used in the plot for the flare duration versus the spot area in Harra et al. (2016, Figure 5). In the present work, the sample number of the on-disk events is expanded to 51 , i.e., by a factor of 2.5 , which contains the previous 20 flares.
}

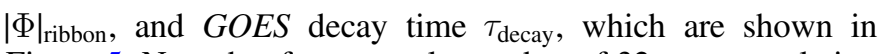
Figure 5. Note that for a sample number of 32, any correlation over 0.45 is significant at $99 \%$ confidence. In this study, we only selected the flares that occurred within $45^{\circ}$ from disk center, which makes the $V_{\mathrm{CME}}$ values rather uncertain. Projection effects due to nonradial motions may also increase the scatter. However, even with such uncertainties, the results show significant higher correlation.

\section{MAGNETIC PATTERNS OF FLARE ZONES}

In flaring ARs, sheared magnetic structures responsible for the flare productions, such as sheared PILs, are probably created by the large-scale flux emergence and the (resultant) relative motions of the sunspots (e.g., Kurokawa 1989). Besides, the geometrical relationship between the sheared PILs and the entire AR may determine the characteristics of the flare events. Therefore, in this section we focus on the creation of sheared PILs in the entire ARs and investigate the flare production in different types of ARs. The details of this characterization are summarized in Figure 6.

The first characterization is the "spot-spot" group, in which a large, long sheared PIL extends across the entire AR between the two major polarities or between the two clusters of sunspots of opposite polarities. Such ARs may naturally harbor large flare ribbons. Among the 11 ARs (21 events) that belong to this category (see bottom row of Figure 6), NOAA AR 11429 produced the strongest (X5.4-class) flare so far in this solar cycle. Based on the numerical simulation of flux emergence, Takasao et al. (2015) suggested the possibility that AR 11429 was created by the emergence of a tightly twisted, kinkunstable flux tube (see, e.g., Tanaka 1991; Linton et al. 1996; Fan et al. 1998). The spot-spot group may also be created by many episodes of flux emergence. 


\section{CME eruptive and non-eruptive}
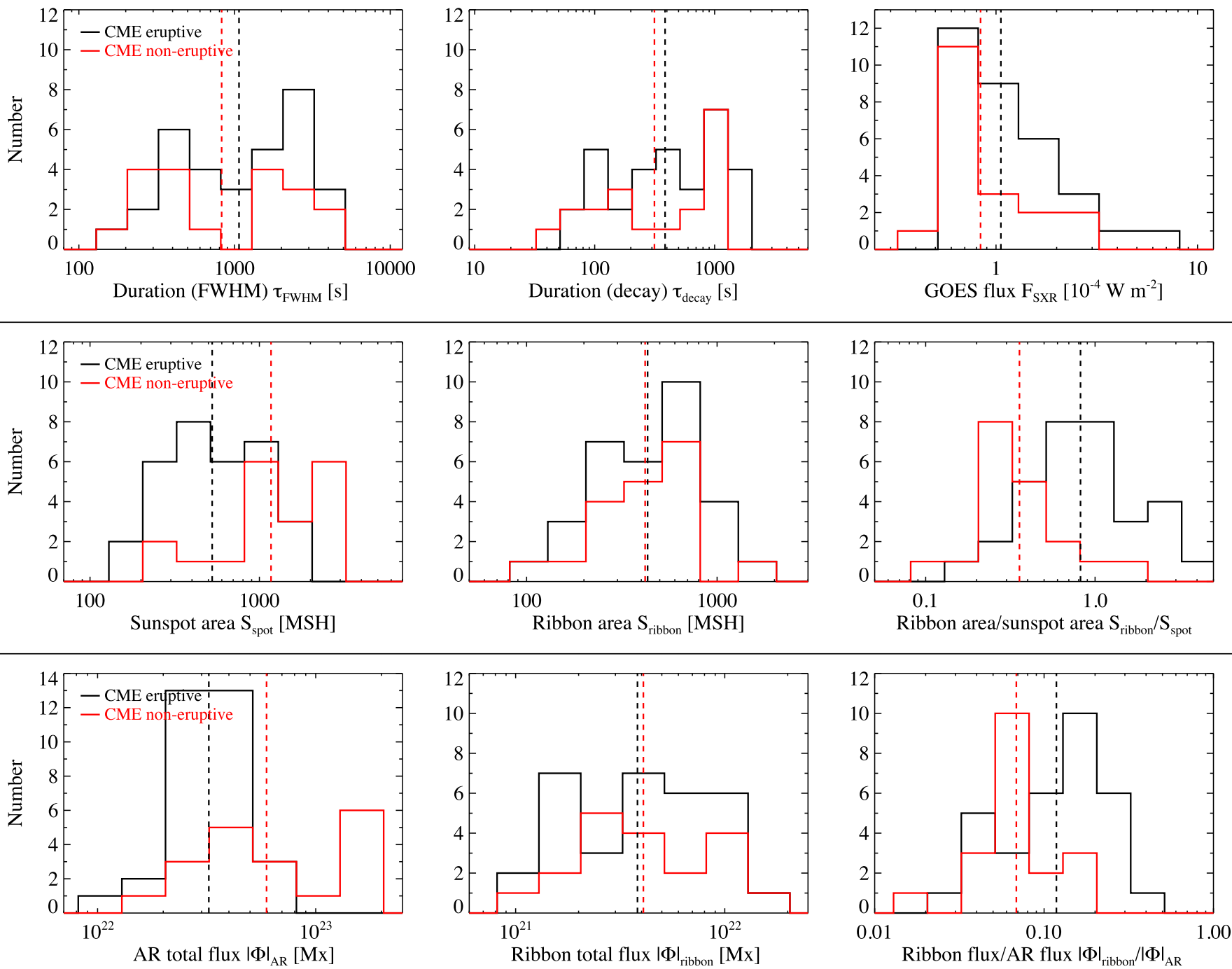

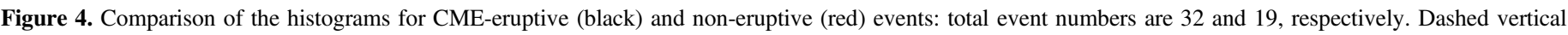

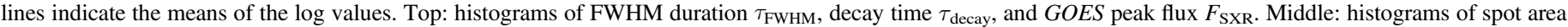
$S_{\text {spot }}$, ribbon area $S_{\text {ribbon, }}$, and their ratio $S_{\text {ribbon }} / S_{\text {spot }}$. Bottom: histograms of AR total magnetic flux $|\Phi|_{\text {AR }}$, ribbon flux $|\Phi|_{\text {ribbon }}$, and their ratio $|\Phi|_{\text {ribbon }} /|\Phi|_{\text {AR }}$.

The second group is that of the "spot-satellite." A total of 25 events from 15 ARs belong to this category. Here, newly emerging, often minor, magnetic flux appears just next to one of the preexisting main polarities and creates a compact PIL between the main and satellite spots. Such a close emergence of satellite spots hints that the satellite spots are connected to the main polarity below the surface as a parasite tube, like that illustrated in Figure 6. Or perhaps the satellite spots are from an independent minor flux tube, which is floating in the convection zone and trapped by the main tube that rises through the interior. The "best-observed" X1.0-class flare of AR 12017 (event \#31; Kleint et al. 2015) falls into this category.

Then, the "quadrupole" group follows these two majorities (three events, two ARs). In this group, two opposite polarities from different emerging bipoles collide with each other, show shear motion, and create a sheared PIL in between. By comparing flux emergence simulation and observational data, Toriumi et al. (2014b) obtained a suggestion that AR 11158 is created from a single flux tube that emerges at the two locations (Fang \& Fan 2015).
The last group, "inter-AR," is of the flares produced on the PIL formed between two apparently independent ARs (two events from different AR pairs). The clearest example is the X1.2-class flare (event \#29; Möstl et al. 2015), which occurred between AR 11944 and the decayed AR 11943. This category resembles the quadrupole events. However, we here divide these two groups by whether the flare occurred between the polarities that belong to a single NOAA-numbered AR or between the polarities of independent ARs with different NOAA numbers, since this categorization may imply whether a mutual (subsurface) magnetic connectivity exists or not. In fact, neither of the inter-AR events have a $\delta$-configuration at the flaring site. Thus, this group reminds us of the eruption of a quiescent filament, which is created in the quiet Sun between extended AR remnants, probably with the support of shear flows caused by the differential rotation (Mackay et al. 2010). Perhaps the inter-AR events occupy an intermediate position between the flares from ARs and those of quiescent filament eruptions.

In reviewing these four patterns identified in flaring regions, we note that the spot-spot group may possess larger flare ribbons since the flares of this group are likely to occur above the 
Scatter plots with strongest correlations
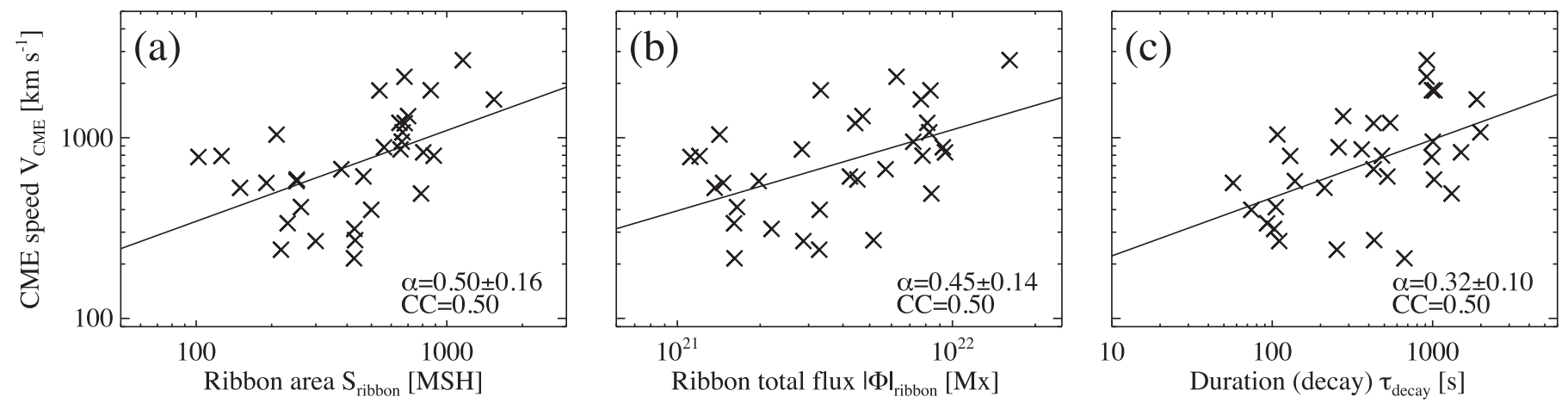

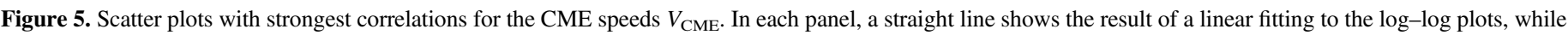
power-law index $\alpha$ and correlation coefficient $\mathrm{CC}$ are shown at the bottom right.

extended sheared PILs across the entire ARs. Conversely, the spot-satellite events are expected to have smaller ribbons. The top row of Figure 7 clearly shows the above trends. Here, the spotspot events have larger ribbon distance, ribbon flux, and ribbon area, while the spot-satellite flares have smaller values. The log averages of the above parameters for the spot-spot and spotsatellite groups are $d_{\text {ribbon }}=51.0$ and $12.9 \mathrm{Mm}$ (difference $=119 \%), \quad|\Phi|_{\text {ribbon }}=7.9 \times 10^{21}$ and $2.3 \times 10^{21} \mathrm{Mx}$ $(112 \%)$, and $S_{\text {ribbon }}=715$ and $277 \mathrm{MSH}(88 \%)$. The quadrupole and inter-AR values generally sit between the two major groups.

Then, through the statistical relations (1)-(3), the spot-spot events have longer GOES durations, and the spot-satellite ones are shorter (middle row of Figure 7). Again, the quadrupole and inter-AR events are in the intermediate positions. As seen from the bottom row of this figure, the spot-spot events have the FWHM durations of $\gtrsim 1000 \mathrm{~s}$, the spot-satellite events $\lesssim 1000 \mathrm{~s}$.

Similar trends are obtained for the $e$-folding decay time through relations (4)-(6). The critical value dividing the two regimes is $\tau_{\text {decay }} \sim 200$ s. However, the GOES peak flux does not show a prominent contrast between the distributions of the spot-spot and spot-satellite events: log-mean values are $1.1 \times 10^{-4}$ and $0.86 \times 10^{-4} \mathrm{~W} \mathrm{~m}^{-2}$, respectively (difference $=27 \%$ ).

These results lead us to the conclusion that the structural differences of the flaring ARs determine the size of the sheared PILs and thus of the flare ribbons, which dictate the flare durations. On the other hand, the GOES flux has much weaker relation to the structural differences, which we hypothesize to reflect that factors other than only geometry are involved in setting the total energy and intensity profile of a flare.

The fractions of the CME-eruptive events for the spot-spot, spot-satellite, quadrupole, and inter-AR events are 57\% (12 in 21 events), 64\% (=16/25), 67\% (=2/3), and $100 \%(=2 / 2)$, respectively. Therefore, the spot-spot events are less likely to be CME-eruptive than the spot-satellite events. This result is well in line with the discussions in the previous sections that a strong overlying arcade, which is likely to exist in a spot-spot $\mathrm{AR}$, prohibits the CME eruption. However, because of the small sample numbers, it is difficult to make any firm conclusion on the quadrupole and inter-AR events.

\section{POSSIBILITIES OF HISTORICAL DATA ANALYSIS}

Figure 8 shows perhaps the largest-ever-imaged sunspotrelated flare ribbons. This sunspot group, numbered 14585 by the Royal Greenwich Observatory (RGO) and 8129 by the Mount Wilson Observatory, produced a great flare on 1946
July 25 (flare importance 3+; Ellison 1949), in modern usage SOL1946-07-25. In the list of sunspot groups with largest areas maintained at NAOJ, ${ }^{10}$ RGO 14585 ranks fourth. According to Dodson \& Hedeman (1949) and RGO reports, this region had a spot size of $4279 \mathrm{MSH}$ that day with $\beta \gamma$-configuration. Ellison (1946) observed in $\mathrm{H} \alpha$ and many other lines and reported that the great flare continued for several hours. It started before 16:15 UT and reached its maximum intensity around 16:30 UT. By 17:30 UT, a bright emission had increased to $2500 \mathrm{MSH}$ in area, accompanied by a filament of $550 \mathrm{Mm}$ in length. His observation continued at least until 18:10 UT. The flare caused a great geomagnetic storm $26.5 \mathrm{hr}$ later and even triggered a ground-level enhancement (GLE; Forbush 1946; Neher \& Roesch 1948). This region repeatedly produced flare eruptions (Dodson \& Hedeman 1949).

As seen from Figure 8, this region is composed of a number of spots, i.e., highly fragmented. On the other hand, it exhibits a giant flare ribbon that extends over the entire region. In fact, our measurements of the spot size in Ca II K1v, $S_{\text {spot }}$, and ribbon size in $\mathrm{H} \alpha, S_{\text {ribbon, }}$ are 4200 and $3570 \mathrm{MSH}$ (projectioncorrected values), respectively. Here, $S_{\text {ribbon }}$ might be underestimated because the ribbon possibly expanded more in the later phase, and thus the area ratio, $S_{\text {ribbon }} / S_{\text {spot }}$, is at least $85 \%$, which indicates that a considerable fraction of RGO 14585 was involved in the flare production.

We can place this region in the context of our present sample through the relationship in Figure 9, which shows the scatter plot of AR total flux $|\Phi|_{\mathrm{AR}}$ versus spot area $S_{\text {spot }}$ for the $51 \geqslant$ M5-class events that we analyzed in Section 3. Note that $S_{\text {spot }}$ indicates the total area of umbrae and penumbrae, i.e., the sunspot area, rather than the area of the entire AR. The linear fitting to this $\log -\log$ plot provides the relation of

$$
\log |\Phi|_{\mathrm{AR}}=(0.74 \pm 0.04) \log S_{\text {spot }}+(20.5 \pm 0.13) .
$$

Using this equation, the measured spot area of $4200 \mathrm{MSH}$ on July 25 yields a flux of $1.5 \times 10^{23} \mathrm{Mx}$, which is comparable to the maximum of our $\geqslant$ M5 data set, $(1.4-1.7) \times 10^{23} \mathrm{Mx}$ of AR 12192. Although this region appeared before the $\delta$ classification was introduced by Künzel (1960), this region is likely to possess a $\delta$-configuration since the long flare ribbons lie in the middle of the spots that share common penumbrae. For the same reason, we can categorize this region as "spot-spot."

\footnotetext{
${ }^{10}$ http://solarwww.mtk.nao.ac.jp/en/bigspots.html
} 
Spot-spot
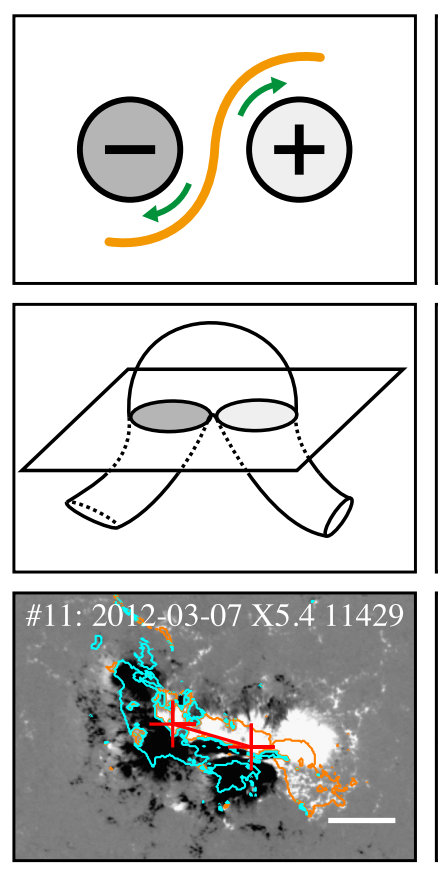

NOAA ARs

$11429,11520,11944$

(\#28), 12036, 12158,

$12192,12205,12222$,

$12241,12242,12371$
Spot-satellite
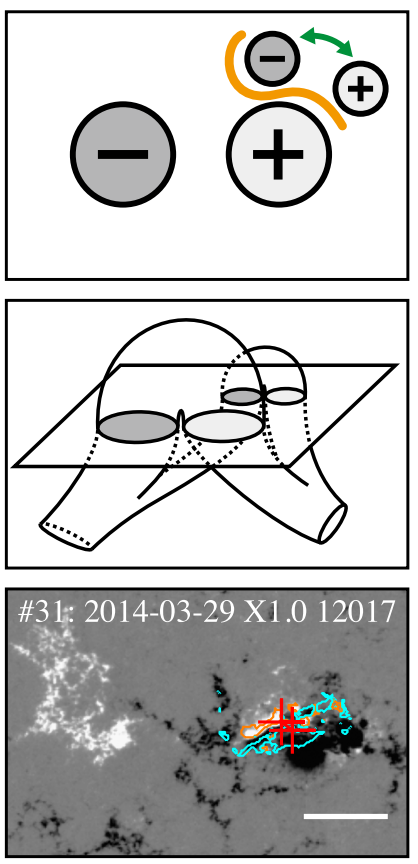

NOAA ARs

$11166,11261,11283$, $11402,11476,11515$, $11719,11877,11884$, $11890,11967,12017$, $12297,12403,12422$
Quadrupole
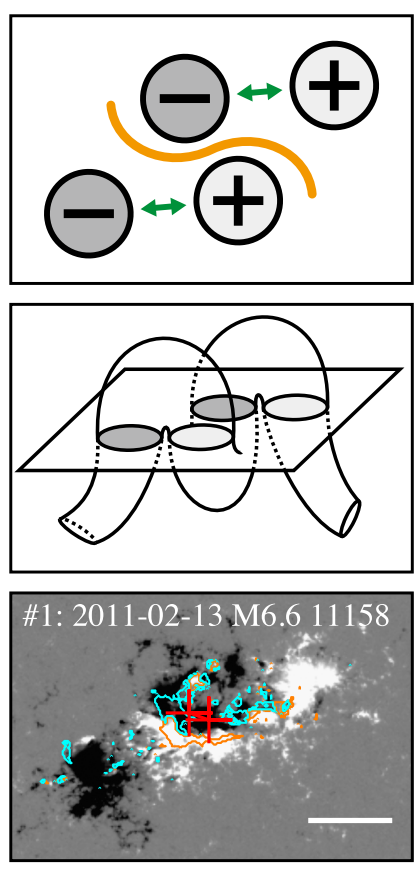

NOAA ARs

11158,11936
Inter-AR
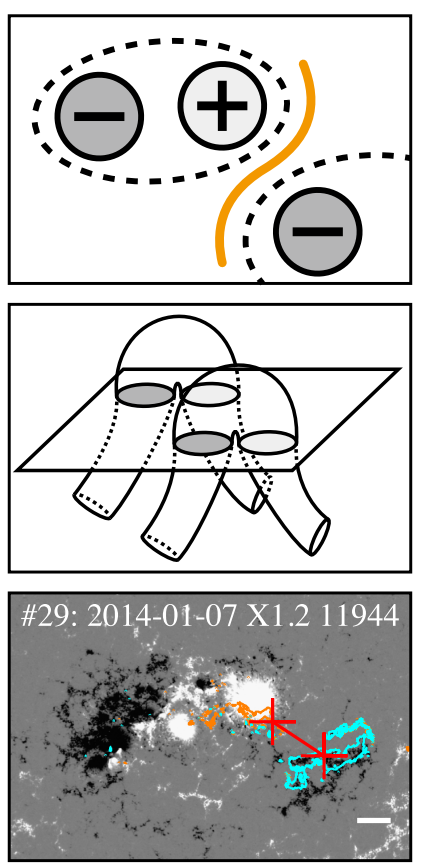

NOAA ARs

11944 (\#29), 12173

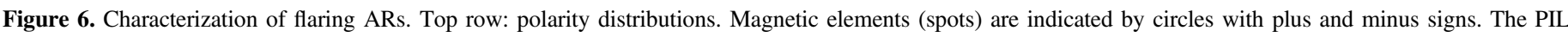

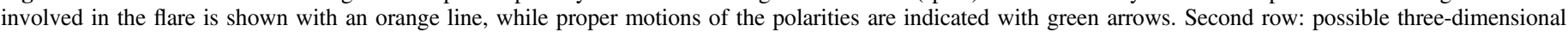

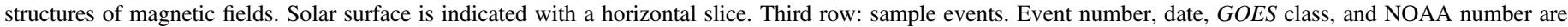

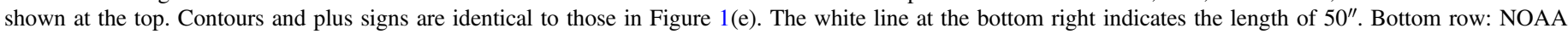
numbers of the corresponding ARs. Event numbers (Table 1) are also shown for AR 11944 to distinguish its two flare events.

In addition, from the $\mathrm{H} \alpha$ image, we estimated the ribbon distance $d_{\text {ribbon. }}$. We here took the two largest ribbon groups and measured the distance between the centroids; see Figure 8(d). Through Equation (1), the obtained value, $d_{\text {ribbon }}=62 \mathrm{Mm}$, which should also be considered as a lower limit, yields the FWHM duration, $\tau_{\mathrm{FWHM}}$, of $2400 \mathrm{~s}$. The actual duration of the flare event is not clear but may be a few times of this value, say, a few hours. In fact, the observations revealed that the flare continued at least for 110 minutes (Ellison 1946; observed mainly in $\mathrm{H} \alpha$ ).

Furthermore, the large area ratio of this event, $S_{\text {ribbon/ }}$ $S_{\text {spot }} \geqslant 85 \%$, implies the occurrence of a CME (see, e.g., Figure 4). In fact, the great flare caused a geomagnetic storm after $26.5 \mathrm{hr}$ and even a GLE (Forbush 1946), which suggests the existence of a severe disturbance such as a fast CME.

\section{DISCUSSION}

\subsection{Interpretation of the Obtained Relations}

In this study, we have conducted a statistical analysis of 51 solar flares with GOES magnitude $\geqslant$ M5 emanating from 29 ARs, and we have obtained six high-correlation $(|\mathrm{CC}| \geqslant 0.64)$ empirical relations, Equations (1)-(6). They indicate that the durations of the GOES light curves (FWHM duration $\tau_{\text {FWHM }}$ and $e$-folding decay time $\tau_{\text {decay }}$ ) correlate linearly with the flare parameters (ribbon distance $d_{\text {ribbon }}$, ribbon total flux $|\Phi|_{\text {ribbon, }}$, and ribbon area $S_{\text {ribbon }}$ ). If we use $\tau_{\text {flare }}$ to simply denote the flare duration, they can be characterized by

$$
\begin{gathered}
\tau_{\text {flare }} \propto d_{\text {ribbon }}, \\
\tau_{\text {flare }} \propto|\Phi|_{\text {ribbon }},
\end{gathered}
$$

and

$$
\tau_{\text {flare }} \propto S_{\text {ribbon }}
$$

In this section, we discuss the physical interpretations of these relations.

First, what do the flare parameters, $d_{\text {ribbon, }}|\Phi|_{\text {ribbon, and }}$

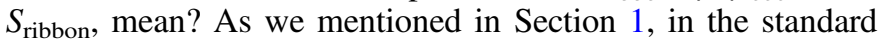
(CSHKP) flare model, the flare ribbons are caused by coronal magnetic energy released into high-energy electrons and thermal conduction. Therefore, we can assume that the ribbons are the footpoints of newly reconnected post-flare loops. Figure 10 shows schematic illustrations of the standard model. As the filament erupts, overlying coronal fields reconnect under the filament, and the post-flare loops and flare ribbons are created. From Figures 10(b) and (c), it is seen that the distance between the two centroids of the ribbon composite, $d_{\text {ribbon }}$, indicates the footpoint separation of the representative postflare loop. If the loop configuration does not differ much for different flare events, the loop half length, $L$, would be 


\section{Flares of different magnetic patterns}
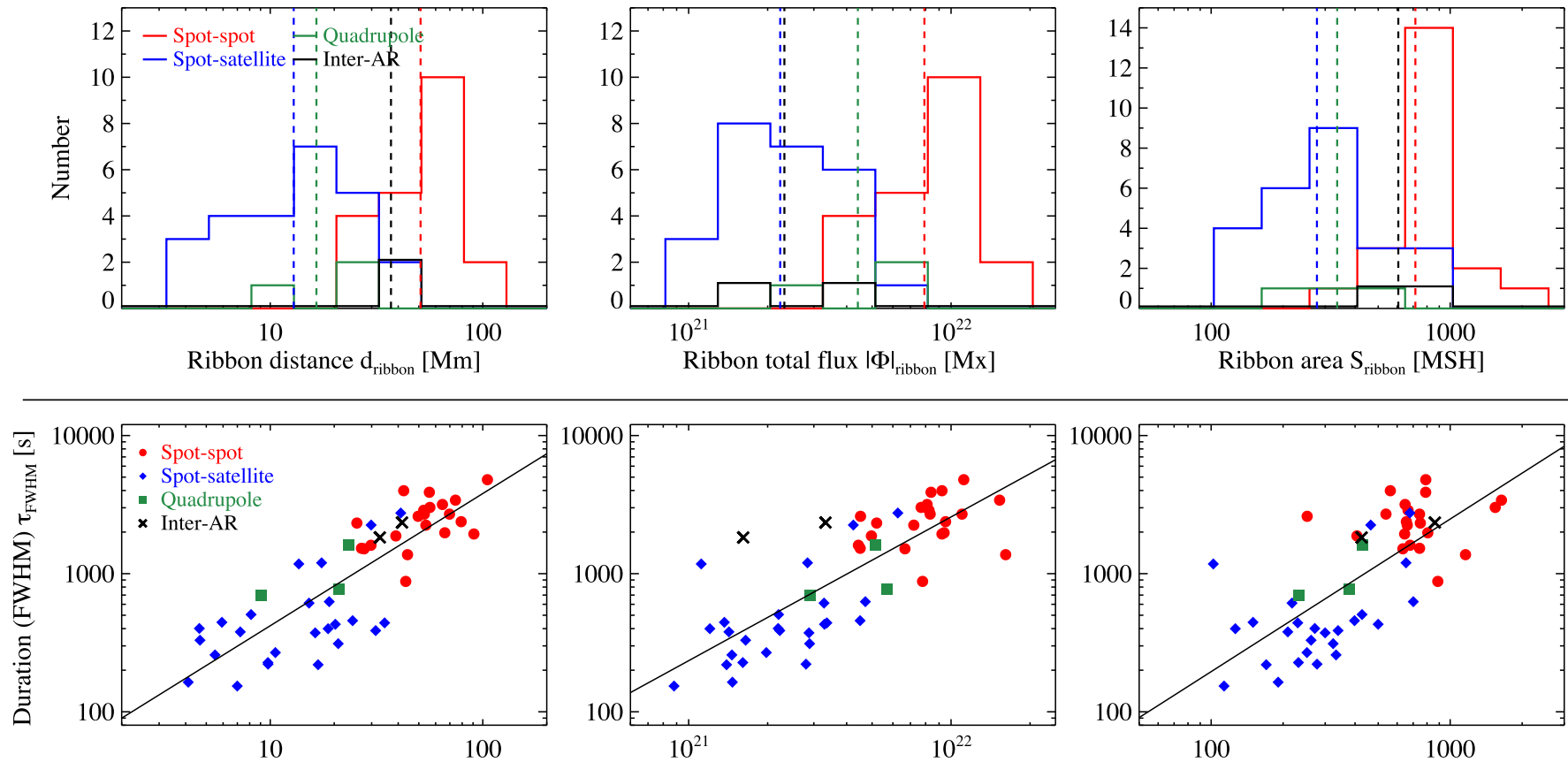

Ribbon distance $\mathrm{d}_{\text {ribbon }}[\mathrm{Mm}]$

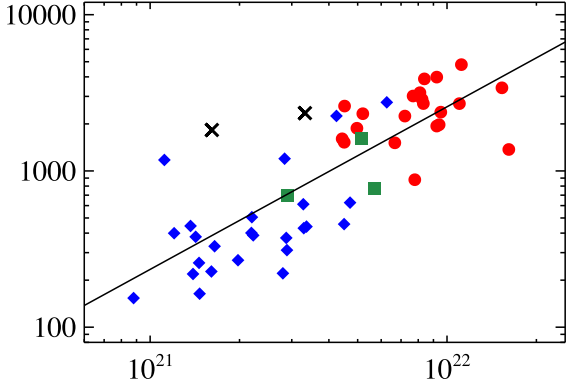

Ribbon total flux $|\Phi|_{\text {ribbon }}[\mathrm{Mx}]$
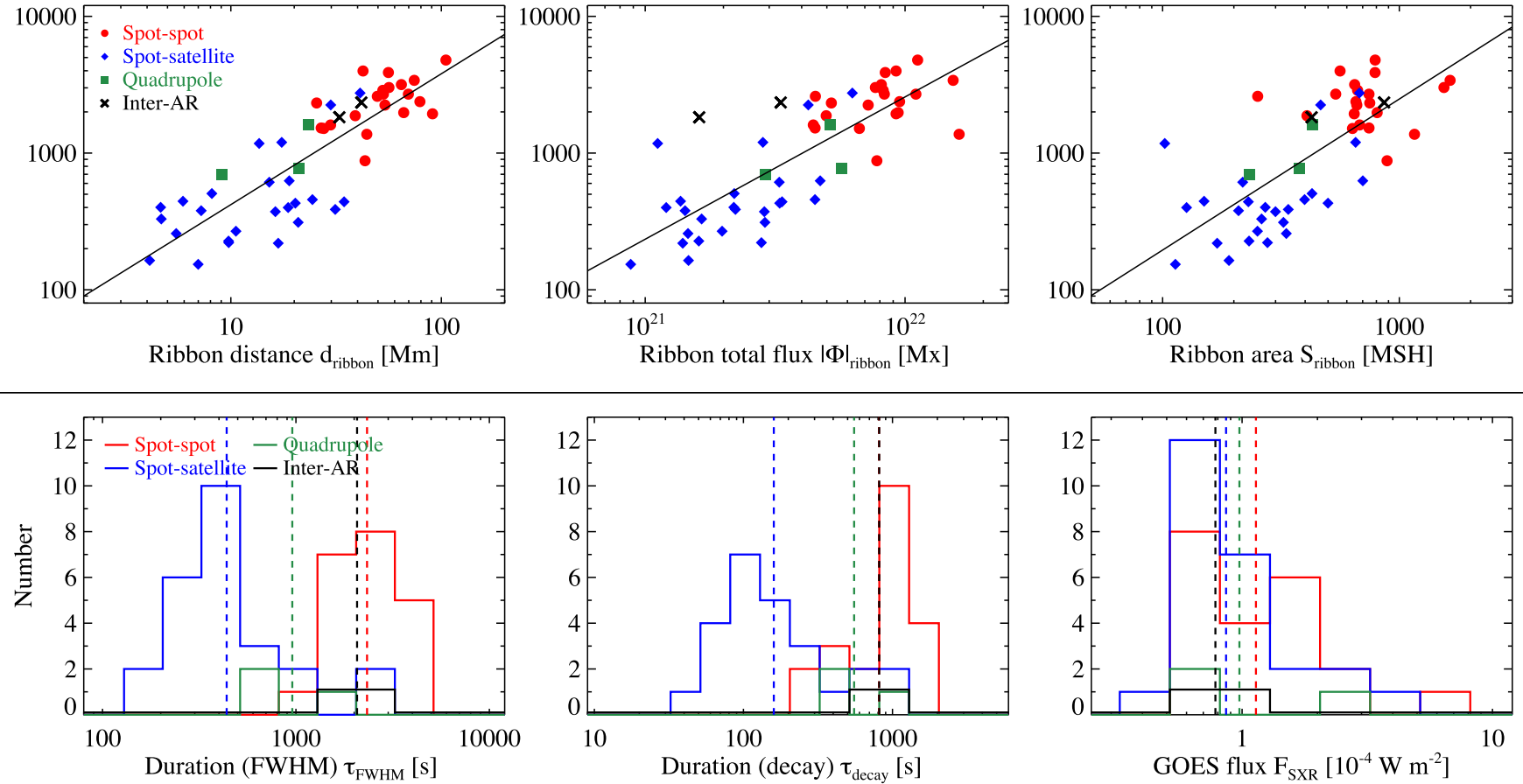

Figure 7. Statistical trends of the flares of different magnetic patterns. Top: histograms for the ribbon distance $d_{\text {ribbon }}$, ribbon flux $|\Phi|_{\text {ribbon }}$, and ribbon area $S_{\text {ribbon }}$ Colors represent the patterns: spot-spot (red), spot-satellite (blue), quadrupole (green), and inter-AR (black). Middle: scatter plots of the flare duration $\tau_{\text {FWHM }}$ vs. $d_{\text {ribbon }},|\Phi|_{\text {ribbon }}$, and $S_{\text {ribbon }}$, i.e., the same as Figures 3(a)-(c) but with different symbols. Straight lines show the fitting results. Bottom: histograms of the FWHM duration $\tau_{\mathrm{FWHM}}, e$-folding decay time $\tau_{\text {decay }}$, and GOES peak flux $F_{\mathrm{SXR}}$.

proportional to $d_{\text {ribbon }}$, i.e., $L \propto d_{\text {ribbon }}$. Meanwhile, $|\Phi|_{\text {ribbon }}$ indicates the total magnetic flux in the ribbon composites, identifiable with the flux involved in the flare reconnection, whereas $S_{\text {ribbon }}$ is the total area of the composite.

For explaining the first relation, $\tau_{\text {flare }} \propto d_{\text {ribbon }}$, we here simply assume that the duration of the flares, especially the evolutionary phase when the ribbon expansion occurs (see Section 2.2), is comparable to the reconnection timescale, i.e., $\tau_{\text {flare }} \sim \tau_{\text {rec }}$. This timescale is roughly estimated as $\tau_{\text {rec }} \sim L / V_{\text {in }}$, where $V_{\text {in }}$ is the velocity of pre-reconnection magnetic fields flowing into the electric current sheet, and this relation is rewritten as $\tau_{\text {rec }} \sim \tau_{\mathrm{A}} / M_{\mathrm{A}}$, where $\tau_{\mathrm{A}} \equiv L / V_{\mathrm{A}}$ is the Alfvén transit time over the loop, $V_{\mathrm{A}}$ the Alfvén velocity, and $M_{\mathrm{A}}=V_{\text {in }} / V_{\mathrm{A}}$ the Alfvén Mach number. If we assume from Figure 10 (c) that $L \sim d_{\text {ribbon, }}$ we find the proportionality $\tau_{\text {flare }} \sim d_{\text {ribbon }} /\left(V_{\mathrm{A}} M_{\mathrm{A}}\right)$.

It is seen, for example, from Figure 3(a) that $V_{\mathrm{A}} M_{\mathrm{A}}=20-30 \mathrm{~km} \mathrm{~s}^{-1}$, and applying $M_{\mathrm{A}}=0.01-0.1$, the typical values for the Petschek-type reconnection model
(Petschek 1964) obtained from resistive-MHD simulations (e.g., Yokoyama \& Shibata 1997, 1998), one may find that $V_{\mathrm{A}}$ ranges from a few times $10^{2}$ to a few times $10^{3} \mathrm{~km} \mathrm{~s}^{-1}$. Such values can be consistent with the Alfvén speed inferred observationally for the solar corona (e.g., Gopalswamy et al. 2001), though not the core of an AR, indicating that the above estimation is fairly plausible.

However, because the observed characteristics in this study, such as the flare timescales, are the result of the superposition of elementary flare loops, and because each flare loop experiences different stages of thermal processes after the reconnection (i.e., the chromospheric evaporation, conductive cooling, and radiative cooling), which may have different timescales (Reale 2007), it is of high importance to conduct MHD simulations of flare reconnection and post-flare loops including thermodynamic processes in order to explore the essential physics involved in the loop.

Relation (9), $\tau_{\text {flare }} \propto|\Phi|_{\text {ribbon }}$, may be easier to understand: as more magnetic flux is involved, the reconnection processes 


\section{6-07-25 Great flare RGO 14585}

(a) Ca II K1v (07:29)
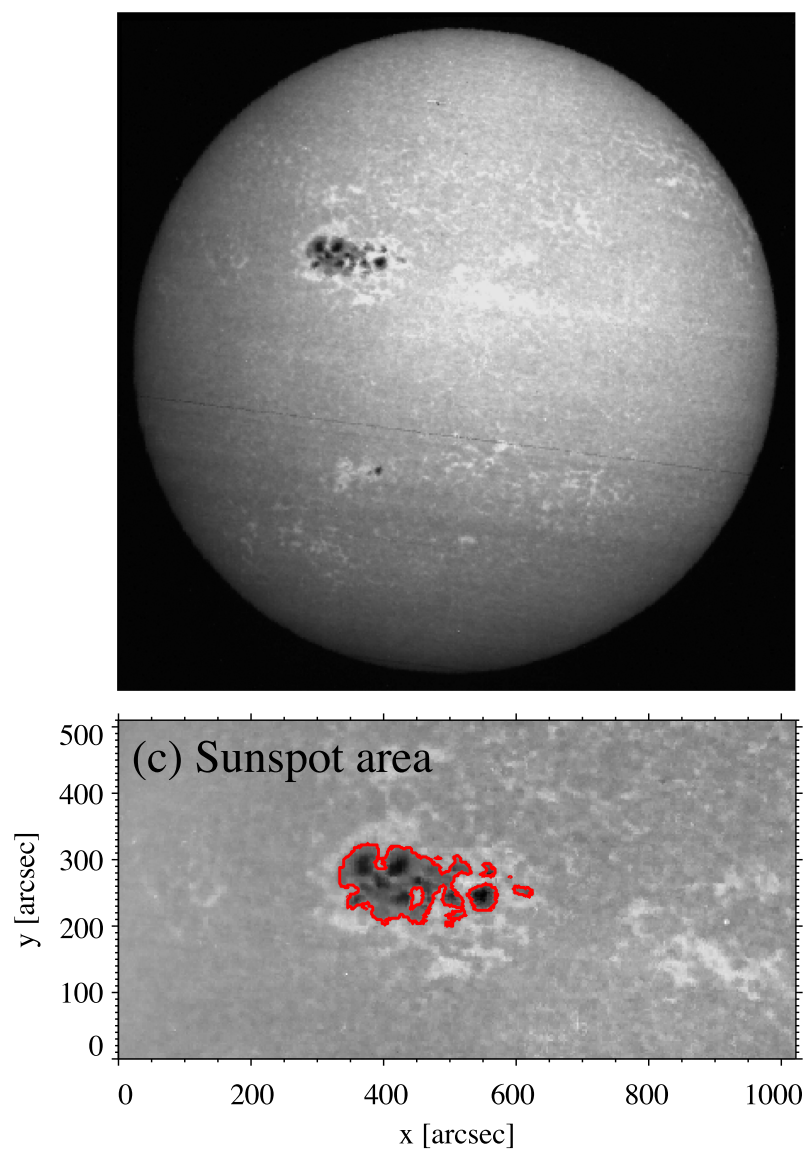

(b) $\mathrm{H} \alpha(17: 32)$
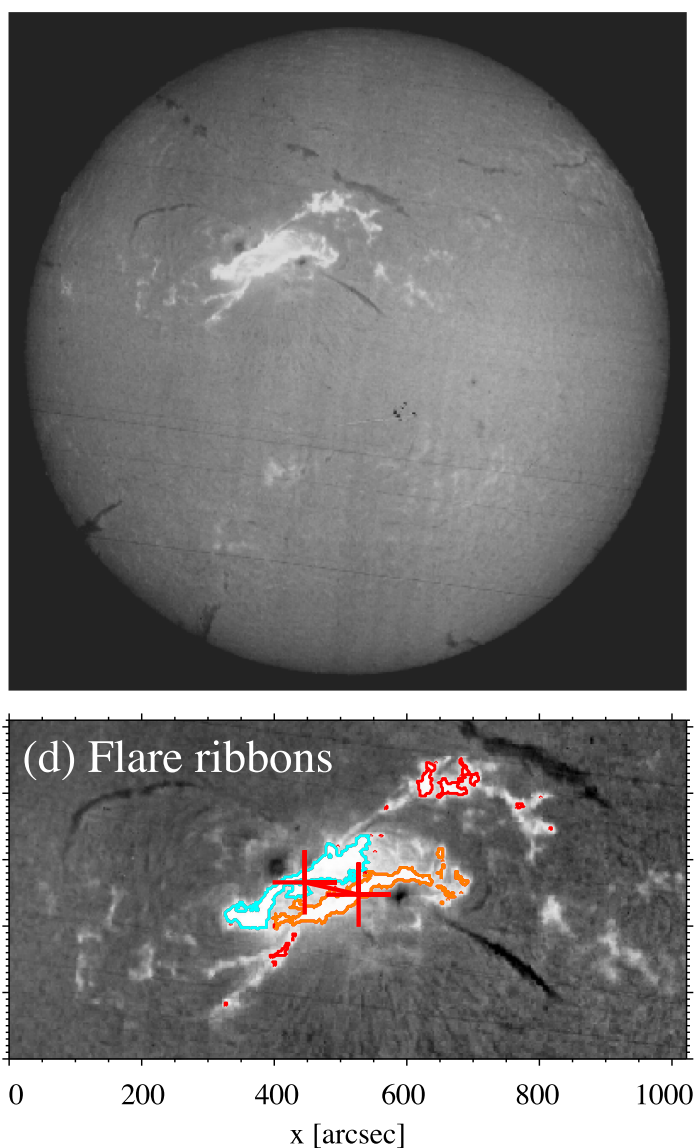

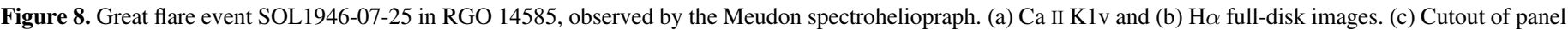

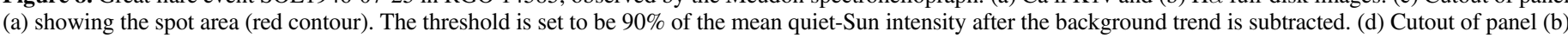

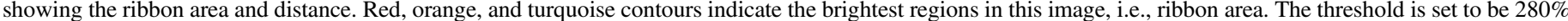

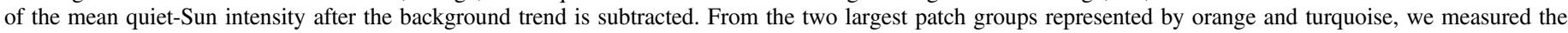
distance between the two centroids, i.e., ribbon distance (red plus signs and a straight line).

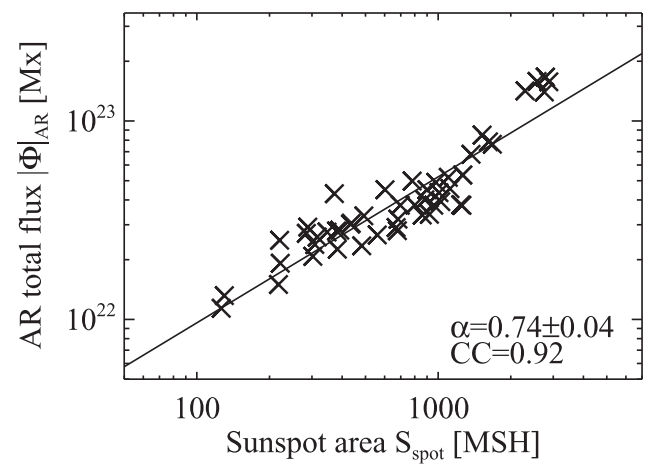

Figure 9. Scatter plot of AR total flux $|\Phi|_{\mathrm{AR}}$ vs. spot area $S_{\text {spot }}$ for the 51 target events. The black straight line is the result of linear fitting to the log-log plot.

continue longer. If the reconnection rate is comparable for various events, the ribbon area also could have a linear proportion, i.e., relation (10), $\tau_{\text {flare }} \propto S_{\text {ribbon }}$.

In any case, the clear correlations between the flare duration and flare parameters (ribbon distance, magnetic flux, and area), especially those of $\tau_{\text {flare }} \propto d_{\text {ribbon }}$, strongly point to the physical connections underlying them. For example, recently it has been suggested that the impulsive events with shorter ribbon distance, $d_{\text {ribbon }}$, yield more intense white-light flares (K. Watanabe et al. 2016, in preparation). This may imply that the loop physics of compact coronal loops, with smaller $L$, corresponds to more intense energy release deeper in the photosphere. We may utilize this relation in the opposite manner. The observation of flare durations may allow us to investigate the physical states of the reconnected loops, such as those of unresolved stellar flares (e.g., Mullan et al. 2006).

\subsection{Emergence, Flares, and Superflares}

One of the important lessons we have learned is that major flares are produced from various types of ARs. X-class events are produced not only from the classical $\delta$-spots, such as those classified by Zirin \& Liggett (1987), or "spot-spot," "spotsatellite," and "quadrupole" in this study, but even from the PILs between separated, independent ARs with no $\delta$-configurations, i.e., "inter-AR," like the X1.2 event from AR 11944 and AR 11943 (event \#29).

Also, the fraction of the region that is involved in the flare reconnection in a single AR differs substantially. The area of the ribbon composite normalized by the spot area, $S_{\text {ribbon }} / S_{\text {spot }}$, ranges from $9.0 \%$ to $300 \%$ for the analyzed $\geqslant$ M5 flares (except 


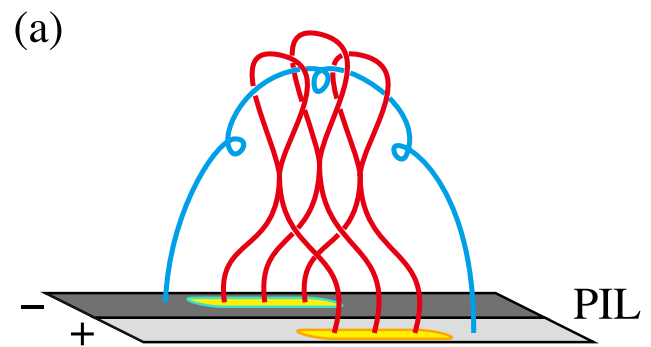

(b)

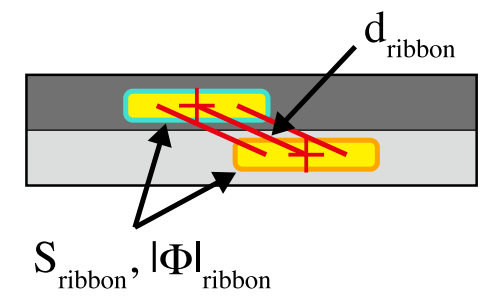

(c)

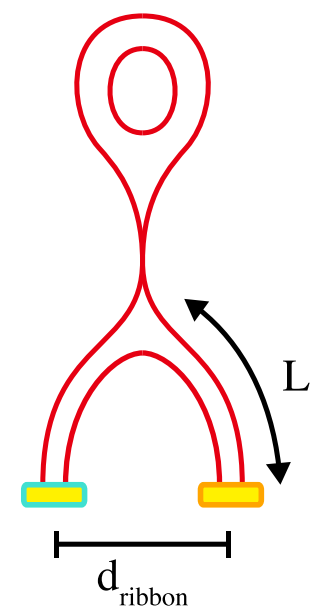

Figure 10. Schematic illustrations of the standard flare model. (a) The filament (cyan), or flux rope, above the PIL between positive $(+)$ and negative $(-)$ polarities erupts, and overlying coronal magnetic fields (red) reconnect under the ascending filament. As a result, flare ribbons (yellow regions outlined by orange and turquoise lines) are created in the chromosphere. (b) Top view of panel (a). Ribbon distance $d_{\text {ribbon }}$, ribbon area $S_{\text {ribbon }}$, and ribbon total flux $|\Phi|_{\text {ribbon }}$ are indicated. Red plus signs show the centroids of the ribbons. (c) Side view of panel (a). Half length of the reconnected (post-flare) loops $L$ is indicated along with the ribbon distance $d_{\text {ribbon }}$.

for the two inter-AR events), while the ribbon flux normalized by AR flux, $|\Phi|_{\text {ribbon }} /|\Phi|_{\mathrm{AR}}$, ranges from $1.6 \%$ to $43 \%$.

Therefore, we need a systematic survey using flux emergence simulations to model these types of ARs (Toriumi et al. 2014b; Fang \& Fan 2015; Takasao et al. 2015; Chatterjee et al. 2016) and investigate their formation processes, as well as the storage of magnetic energy (amount, place, etc.). In Figure 4 , we found that $S_{\text {ribbon }} / S_{\text {spot }}$ and $|\Phi|_{\text {ribbon }} /|\Phi|_{\mathrm{AR}}$ are larger for the CME-eruptive events, which may indicate the importance of the relative magnetic structure of the flaring region and the entire AR. Thus, numerical experiments on flux emergence and flare AR formation are necessary also for the investigation on the CME productions.

From the statistical analysis of the stellar flares obtained by the Kepler space telescope, Maehara et al. (2012) suggested that superflares with energy of $10^{34}$ erg occur once every 800 $\mathrm{yr}$ on the Sun-like stars (slowly rotating G-type main-sequence stars). Shibata et al. (2013) showed through order-of-magnitude estimations that in typical solar dynamo models, it may be possible to generate a large sunspot with a total flux of $2 \times 10^{23} \mathrm{Mx}$, which accounts for the flare of $10^{34} \mathrm{erg}$, within one solar cycle period. On the other hand, Aulanier et al. (2013) argued that superflares of $10^{34} \mathrm{erg}$ are unrealistic for observed solar conditions because of the fragmentation of magnetic flux in an AR: all large sunspot groups are highly fragmented, i.e., composed of many flux emergence events, and thus magnetic shear tends to be localized. Therefore, only parts of the sunspots might be involved in the flare reconnection process (see also Schrijver et al. 2012).

However, as we saw in Section 5, even one of the largest, highly fragmented sunspot groups such as RGO 14585 could spout a flare eruption leaving AR-sized, gigantic flare ribbons, which may point to the possibility that even larger ARs could occur and cause a superflare. The largest sunspot group since the nineteenth century, RGO 14886, recorded a maximum spot area of $6132 \mathrm{MSH}$ on 1947 April 8 (see Figure 3 of Aulanier et al. 2013). From Equation (7), we estimate its total flux to be $2.0 \times 10^{23} \mathrm{Mx}$. Therefore, considering the two factors that one of the largest ARs produced the AR-scale eruption and an AR of $2 \times 10^{23} \mathrm{Mx}$ is likely to have existed, we cannot completely rule out the possibility that an AR of $2 \times 10^{23}$ Mx produces AR-scale eruptions. We will then estimate the flare energy in the next section.

\subsection{Estimation of Flare Energy}

The magnetic energy that we discuss in this section is given using flare parameters as

$$
\begin{aligned}
& E_{\text {mag }} \sim \frac{B^{2}}{8 \pi} V_{\text {mag }} \sim \frac{|\bar{B}|_{\text {ribbon }}^{2}}{4 \pi} S_{\text {ribbon }} d_{\text {ribbon }} \\
& \sim 4.3 \times 10^{32}\left(\frac{|\bar{B}|_{\text {ribbon }}}{325 \mathrm{G}}\right)^{2}\left(\frac{S_{\text {ribbon }}}{519 \mathrm{MSH}}\right)\left(\frac{d_{\text {ribbon }}}{32.9 \mathrm{Mm}}\right) \mathrm{erg},
\end{aligned}
$$

where $V_{\text {mag }}$ is the volume of magnetic fields involved in the flare reconnection. We assume here that $V_{\text {mag }} \sim 2 S_{\text {ribbon }} L$, where $L$ is the half length of the reconnected loop (Figure 10), and that $L \sim d_{\text {ribbon. }}$. The parameters used in this equation for deriving the typical value are the means from the 51 analyzed events, and the estimated magnetic energy ranges from $9.2 \times 10^{30}$ to $4.4 \times 10^{33} \mathrm{erg}$.

The magnetic energy given by Equation (11) may provide better estimates for the flare energy, $E_{\text {flare }} \sim f E_{\text {mag }}$, where $f$ is the fraction of the magnetic energy that is released in the flare event, compared to another expression (e.g., Maehara et al. 2012; Aulanier et al. 2013; Shibata et al. 2013):

$$
\begin{aligned}
E_{\text {mag }} & \sim \frac{B^{2}}{8 \pi} V_{\text {mag }} \sim \frac{\mid \overline{B \mid}}{8 \pi} S_{\text {spot }}^{3 / 2} \\
& \sim 2.9 \times 10^{33}\left(\frac{|\bar{B}|_{\mathrm{AR}}}{688 \mathrm{G}}\right)^{2}\left(\frac{S_{\mathrm{spot}}}{954 \mathrm{MSH}}\right)^{3 / 2} \mathrm{erg} .
\end{aligned}
$$

For the great flare event of RGO 14585 (SOL1946-07-25), from Equation (11) with $\bar{B}_{\text {ribbon }} \sim 384 \mathrm{G}$ (mean of the spotspot events), $S_{\text {ribbon }} \sim 3570 \mathrm{MSH}$, and $d_{\text {ribbon }} \sim 62 \mathrm{Mm}$, the energy estimate becomes $8 \times 10^{33} \mathrm{erg}$. If we suppose the situation that the largest sunspot group RGO 14886 $\left(S_{\text {spot }}=6132 \mathrm{MSH}\right.$ on 1947 April 8) causes a whole-AR- 


\section{Time versus magnetic energy}
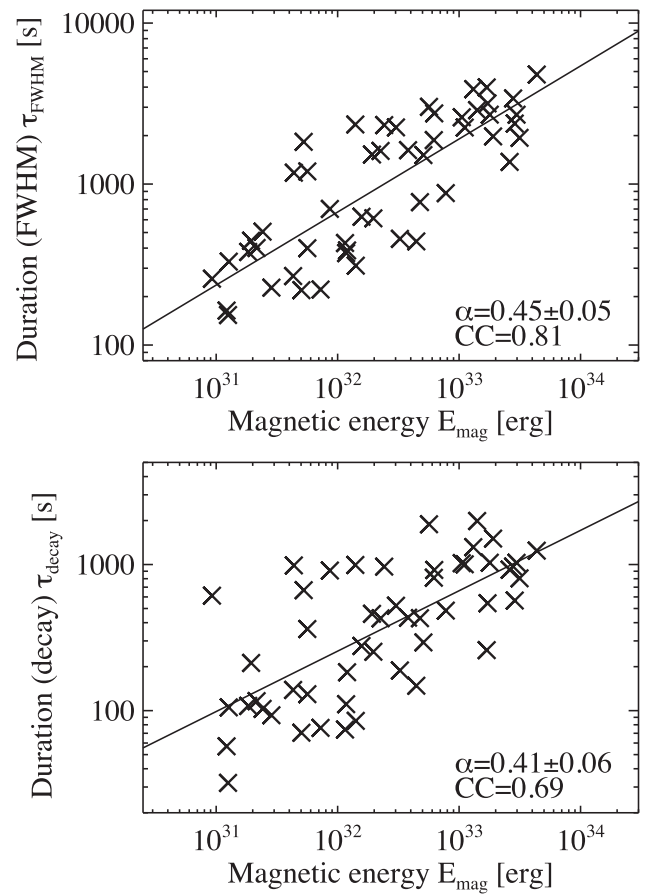

Figure 11. Scatter plots of flare timescales $\tau_{\text {FWHM }}$ and $\tau_{\text {decay }}$ vs. magnetic energy $E_{\text {mag }}$ (Equation (11)). Straight lines show the results of linear fitting to the $\log -\log$ plots.

scale eruption like the 1946 event, which may not be very unrealistic, using the values of $\overline{|B|}$ ribbon $\sim 384 \mathrm{G}$, $S_{\text {ribbon }} \sim 5210 \mathrm{MSH}$ (assuming an area ratio, $S_{\text {ribbon }} / S_{\text {spot }}$, of $85 \%$ ), and $d_{\text {ribbon }}$ being, say, $80 \mathrm{Mm}$, the estimated magnetic energy amounts to $1.5 \times 10^{34} \mathrm{erg}$. Although what fraction is converted to the flare energy is not clear, the above results indicate the possibility that the flare energy of such gigantic ARs may be up to of the order of $10^{34} \mathrm{erg}$.

Figure 11 compares the timescales of the flare, $\tau_{\text {flare, }}$ and the magnetic energy given by Equation (11), $E_{\text {mag }}$. The scatter plots show the proportionalities of $\tau_{\mathrm{FWHM}} \propto E_{\mathrm{mag}}^{0.45} \pm 0.05$ (correlation coefficient $\mathrm{CC}=0.81)$ and $\tau_{\text {decay }} \propto E_{\mathrm{mag}}^{0.41 \pm 0.06} \quad(\mathrm{CC}=0.69)$, which is surprisingly consistent with the results of the superflare analysis by Maehara et al. (2015), $\tau_{\text {flare }} \propto E_{\text {flare }}^{0.39 \pm 0.03}$, where $\tau_{\text {flare }}$ and $E_{\text {flare }}$ are the $e$-folding decay time and bolometric energy, respectively. Note that their values are measured from Kepler's photometric data that cover from 4200 to $9000 \AA$, i.e., the optical regime. Maehara et al. (2015) explained this proportionality by combining the two relations, $\tau_{\text {flare }} \sim \tau_{\mathrm{A}} / M_{\mathrm{A}} \sim L / V_{\mathrm{A}} / M_{\mathrm{A}} \propto L$ (Section 6.1) and $E_{\text {flare }} \sim f E_{\text {mag }} \sim f B^{2} L^{3} /(8 \pi) \propto L^{3},{ }^{1,12}$ to give $\tau_{\text {flare }} \propto E_{\text {flare }}^{1 / 3}$. However, from Equations (8), (10), and (11), one can also derive the relation $E_{\text {mag }} \propto S_{\text {ribbon }} d_{\text {ribbon }} \propto \tau_{\text {flare }}^{2}$, which may suggest that the time-energy relation is $\tau_{\text {flare }} \propto E_{\mathrm{mag}}^{1 / 2}$. Still, the consistent proportionalities suggest the existence of the

\footnotetext{
${ }^{11}$ In their order-of-magnitude estimate, Maehara et al. (2015) make the ad hoc assumption that the sunspot field strength $B$ does not vary much for different events and is typically of the same order, $1000 \mathrm{G}$.

12 Many flare analyses are based on the simple assumption that the flare energy $E_{\text {flare }}$ scales with the peak SXR brightness $F_{\text {SXR }}$. However, the low correlations between the SXR brightness and the flare parameters in Table 2 (e.g., $\mathrm{CC}=0.23$ for $F_{\mathrm{SXR}}$ versus $S_{\text {ribbon }}$ ) may indicate that the assumption is not necessarily the case.
}

common physical origin between the solar and stellar flares (Shibata \& Yokoyama 1999, 2002).

\section{CONCLUSION}

In this study, we have examined all $51 \geqslant$ M5.0-class, ondisk ( $\leqslant 45^{\circ}$ from disk center) events, emanating from $29 \mathrm{ARs,}$ in the period of May 2010 to April 2016, i.e., 6 yr from the activity minimum of solar cycle 24 .

Out of the 29 ARs, 24 regions (83\%) showed $\delta$-spot configurations, while three regions violated Hale's polarity rule at the instant of flare occurrence. The 51 flare events consist of $20 \mathrm{X}$ - and $31 \mathrm{M}$-class events.

With the aim to find the physical parameters that dictate the GOES light curves, we systematically surveyed the correlations between GOES parameters (timescales and peak flux) and AR and flare parameters (spot size, ribbon size, etc.) for the 51 events. The strongest correlations were obtained for $\tau_{\text {flare }}$ (i.e., $\tau_{\text {FWHM }}$ and $\tau_{\text {decay }}$ ) versus $d_{\text {ribbon }},|\Phi|_{\text {ribbon }}$, and $S_{\text {ribbon }}$, and all these relations showed approximately linear correlations.

The first relation, $\tau_{\text {flare }} \propto d_{\text {ribbon, }}$, can be explained by assuming that (1) the distance between the ribbon composites in the positive and negative polarities, $d_{\text {ribbon, }}$, represents the length of the reconnected (post-flare) loops, $L$, and (2) the flare duration, $\tau_{\text {flare, }}$ is dominated by the reconnection time, $\tau_{\text {rec }}$, which should be related to the Alfvén transit time over the loop length, $\tau_{\mathrm{A}} \equiv L / V_{\mathrm{A}}$. Then, we obtain the relation $\tau_{\text {flare }} \sim \tau_{\text {rec }} \sim \tau_{\mathrm{A}} / M_{\mathrm{A}} \sim L / V_{\mathrm{A}} / M_{\mathrm{A}} \propto L \propto d_{\text {ribbon. }}$. To further investigate this proportionality with considering the thermal processes, however, we may need the help of loop simulations including thermodynamics, because what we observed is a superposition of elementary flare loops and each flare loop undergoes several stages of thermal processes.

The other two proportionalities, $\tau_{\text {flare }} \propto|\Phi|_{\text {ribbon }}$ and $\tau_{\text {flare }} \propto S_{\text {ribbon }}$, may be easier to understand. The former simply shows that as more magnetic flux is involved, the reconnection processes continue longer. The latter may also be accepted if we assume that the strengths of the field lines are not so different among the events.

The largest-magnitude, or longest-duration, flares do not necessarily produce CMEs. Although this is obvious when considering the perfect example of AR 12192, the statistical analysis clearly shows the general trend that the non-eruptive events have smaller $S_{\text {ribbon }} / S_{\text {spot }}$ and $|\Phi|_{\text {ribbon }} /|\Phi|_{\mathrm{AR}}$, which may indicate that in the non-eruptive regions, the existence of an embedding field inhibits CME eruption. Therefore, we can speculate that the relative structural relation between the flaring region and the entire $A R$ is a key to determining whether the flare becomes eruptive or not.

Most of the 51 flare events under study originated from the interiors of ARs. Only two events are not from inside the ARs but from the boundaries between separated, independent ARs. The first group can be subdivided into three categories, "spotspot," "spot-satellite," and "quadrupole." The latter, the "interAR" group, shows us that high-M or even X-class events can be produced without strong-field, high-gradient PILs. The representative event may be the X1.2 flare from between AR 11944 and AR 11943. Several scenarios were suggested in this paper to model the formation of the above ARs. These should be examined through systematic survey using flux emergence simulations, which we shall leave for future research.

The historical record of a gigantic sunspot group, RGO 14585, allows us to know that even the largest, fragmented 
ARs can produce massive flare eruptions with AR-sized flare ribbons. The estimation of $d_{\text {ribbon }}$ and $S_{\text {ribbon }} / S_{\text {spot }}$ suggests that the great flare of RGO 14585 is a long-duration event with a CME eruption, which is in line with the observational facts. Perhaps this event points to the possibility of the eruption of even larger ARs. Estimations suggest that an AR of $2 \times 10^{23}$ $\mathrm{Mx}$ is likely to have existed and that if it is flaring, it could produce superflares with an energy of order of $10^{34} \mathrm{erg}$.

Finally, we found the correlations of $\tau_{\text {flare }} \propto E_{\text {mag }}^{0.4}$, which is well in line with the stellar flare (superflare) observations. This clear consistency favors a common physical background for solar and stellar flares.

The authors are grateful to the anonymous referee for helping us improve the manuscript. The authors thank ISSI for the support of the solar-stellar team. S.T. would like to thank Dr. Takashi Sakurai for fruitful discussion and continuous encouragement. HMI and AIA are instruments on board SDO, a mission for NASA's Living With a Star program. This CME catalog is generated and maintained at the CDAW Data Center by NASA and The Catholic University of America in cooperation with the Naval Research Laboratory. SOHO is a project of international cooperation between ESA and NASA. The historical Meudon spectroheliograph observations were digitalized by I. Bualé and are available in the BASS2000 database. This work was carried out using the data from the SDO HMI/AIA Joint Science Operations Center Data Record Management System and Storage Unit Management System (JSOC DRMS/SUMS). This work was partially supported by JSPS KAKENHI Grant Numbers JP26887046, JP16K17671, and JP15H05814. K.N. acknowledges support from EU FP7 Collaborative Project "Exploitation of Space Data for Innovative Helio- and Asteroseismology" (SPACEINN).

\section{APPENDIX A}

\section{TARGET FLARES AND MEASURED PARAMETERS}

Figure 12 lists the 51 flare events that we analyzed in this paper. Here we show the HMI magnetogram before the flare onset as a background overlaid by the composite flare ribbons. Ribbon distance is shown by a red line connecting the centroids of the ribbons in the positive and negative polarities. In some events, we separate the target $\mathrm{AR}$ from neighboring flux concentrations with thin lines.

Table 3 shows all measured parameters of the 51 flare events: GOES parameters (durations $\tau_{\text {FWHM }}$ and $\tau_{\text {decay }}$ and GOES flux $F_{\mathrm{SXR}}$ ), AR parameters (spot area $S_{\text {spot, }}$, total flux $|\Phi|_{\mathrm{AR}}$, and field strength $|\bar{B}|_{\mathrm{AR}}$ ), flare parameters (ribbon area $S_{\text {ribbon }}$, distance $d_{\text {ribbon }}$, total flux $|\Phi|_{\text {ribbon }}$, and field strength $|\overline{\mid B}|_{\text {ribbon }}$ ), and a CME parameter (CME speed $\left.V_{\mathrm{CME}}\right)$. The maximum, minimum, median, and standard deviation $(\sigma)$ of each parameter are summarized at the bottom of this table.

\section{APPENDIX B \\ STATISTICAL TESTS ON THE CME-ERUPTIVE AND NON-ERUPTIVE DISTRIBUTIONS}

First, we compare the spot areas $S_{\text {spot }}$ for the CME-eruptive and non-eruptive cases. Here we use suffix 1 for eruptive distribution and 2 for non-eruptive group. From Table 3, the two sample distributions are

$$
\begin{aligned}
S_{\text {spot }, 1}= & {[678,391,315,288,284,220,379,1256,1241,} \\
& 960,801,682,1369,218,437,308,979, \\
& 781,602,433,1617,126,347,492,130,372, \\
& 899,1267,382,560,1120,697] \mathrm{MSH}
\end{aligned}
$$

and

$$
\begin{aligned}
S_{\text {spot }, 2}= & {[482,859,303,1103,881,1008,222,} \\
& 1679,1523,2756,2877,2781,2786,2572, \\
& 2293,670,921,1264,1026] \mathrm{MSH}
\end{aligned}
$$

and the numbers of elements are $n_{1}=32$ and $n_{2}=19$. Using Student's $t$-test (Welch's $t$-test), we examine the null hypothesis " $\mu_{1}=\mu_{2}$ " and the alternative hypothesis " $\mu_{1}<\mu_{2}$ " with the one-tailed test, where $\mu_{1}$ and $\mu_{2}$ are the means of the parent populations.

The statistic $t$ is defined as

$$
t=\frac{\overline{X_{1}}-\overline{X_{2}}}{\sqrt{s_{1}^{2} / n_{1}+s_{2}^{2} / n_{2}}},
$$

where $\bar{X}_{1}$ and $\bar{X}_{2}$ are the means of the two sample distributions, $s_{1}^{2}=\sum\left(X_{1 i}-\overline{X_{1}}\right)^{2} /\left(n_{1}-1\right), \quad$ and $\quad s_{2}^{2}=\sum\left(X_{2 i}-\overline{X_{2}}\right)^{2} /$ $\left(n_{2}-1\right)$. From Equations (13) and (14), we obtain $t=-3.732$.

The degree of freedom $\nu$ is approximated as

$$
\nu=\frac{\left(s_{1}^{2} / n_{1}+s_{2}^{2} / n_{2}\right)^{2}}{\frac{\left(s_{1}^{2} / n_{1}\right)^{2}}{n_{1}-1}+\frac{\left(s_{2}^{2} / n_{2}\right)^{2}}{n_{2}-1}}
$$

and is calculated to be $\nu=22.16$, which is rounded to the nearest integer, $\nu^{*}=22$.

The table relating the test statistics and degrees of freedom shows that $t_{0.005}(22)=2.819$ and $t_{0.0005}(22)=3.792$ :

$$
-t_{0.0005}(22)<t<-t_{0.005}(22) \text {. }
$$

Therefore, the significant probability is between 0.0005 and 0.005 and, at the $99.5 \%$ confidence level, the null hypothesis is rejected, and thus we can conclude that the spot areas of the eruptive events are smaller than the non-eruptive events.

Next, we try the case without AR 12192, the largest spot group of the cycle. The spot area without the six values for AR 12192 is

$$
\begin{aligned}
S_{\text {spot }, 2}= & {[482,859,303,1103,881,1008,222,} \\
& 1679,1523,670,921,1264,1026] \mathrm{MSH}
\end{aligned}
$$

and $n_{2}=13$. Then, from Equations (13) and (18), we obtain $t=-1.967$ and $\nu=20.91 \quad\left(\nu^{*}=21\right)$. In this case, $t_{0.05}(21)=1.721$ and $t_{0.025}(21)=2.080$. Then, we find that the probability falls in between 0.025 and 0.05 and that the null hypothesis is rejected still at $95 \%$ confidence.

The AR total fluxes $|\Phi|_{\mathrm{AR}}$ for the eruptive and non-eruptive cases are

$$
\begin{aligned}
|\Phi|_{\mathrm{AR}, 1}= & {[2.8,2.8,2.6,2.9,2.7,2.5,2.8,3.8,3.8,3.8,} \\
& 3.8,3.2,6.8,1.5,3.1,2.4,4.9,5.0,4.5,3.0, \\
& 7.9,1.1,2.8,3.3,1.3,4.3,4.5,5.3,2.3,2.7, \\
& 4.6,3.8] \times 10^{22} \mathrm{Mx}
\end{aligned}
$$



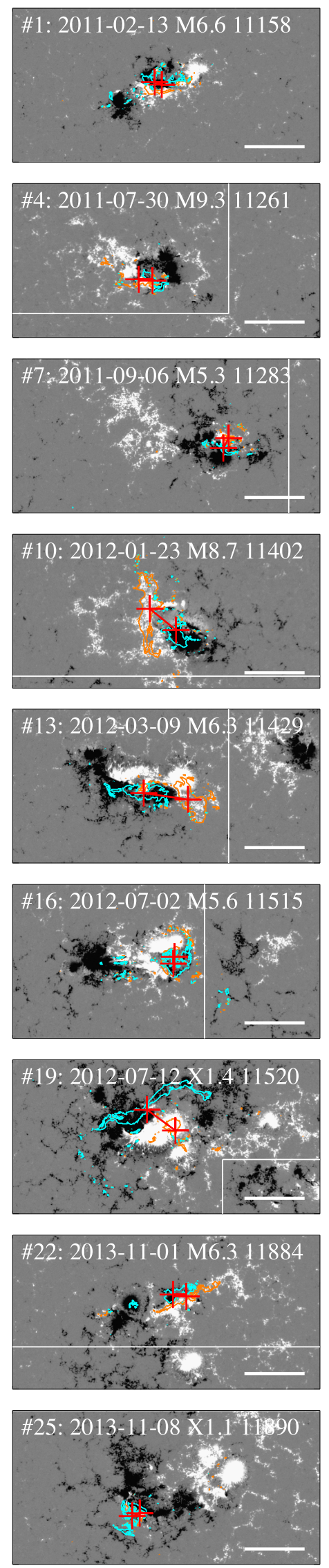
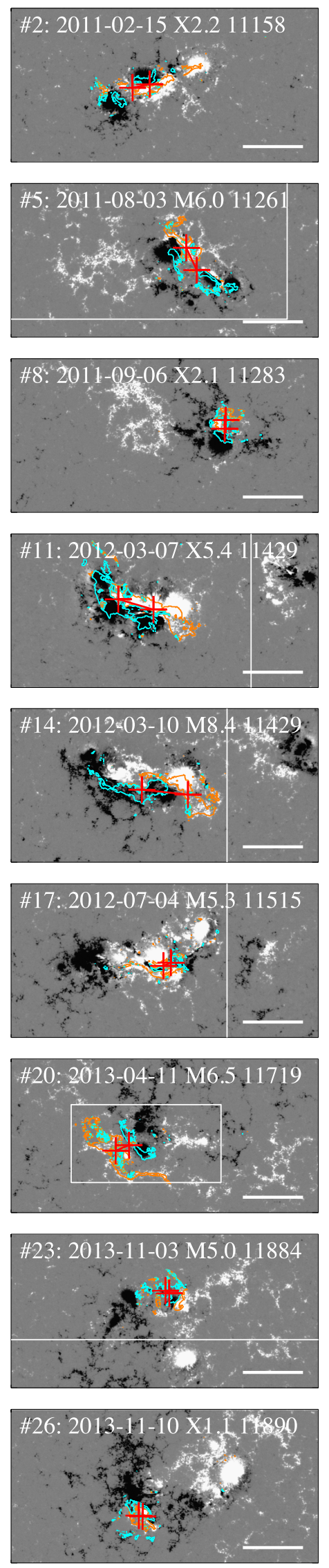
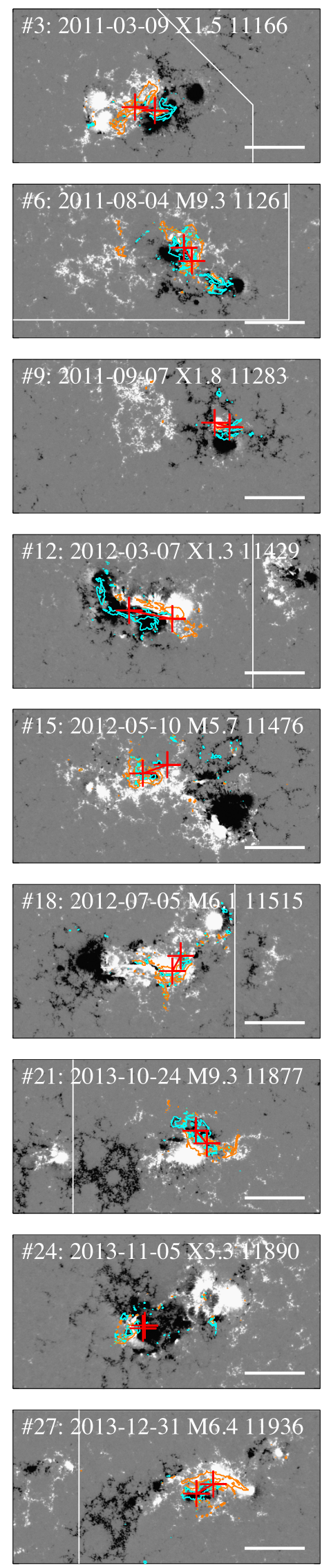

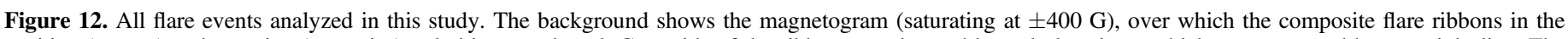

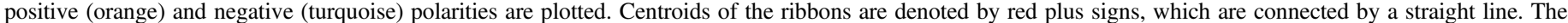
thick white line at the bottom right indicates the length of $100^{\prime \prime}$, while thin white lines separate the target AR from neighboring flux concentrations. 

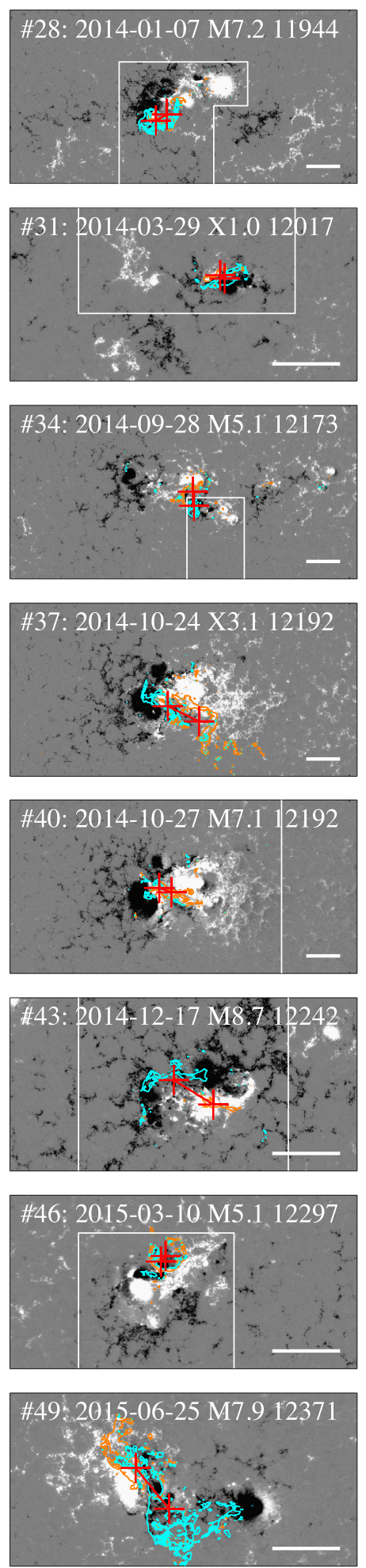
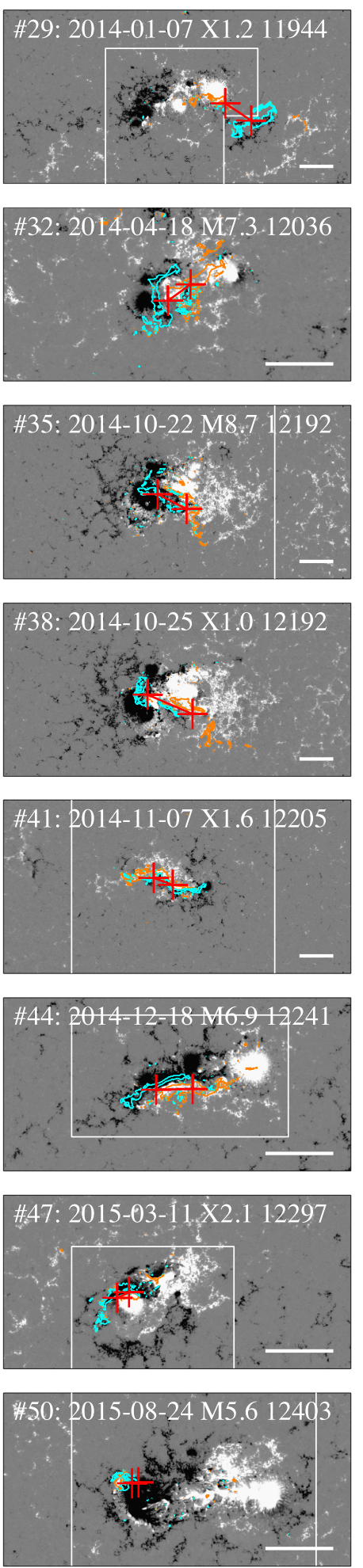

Figure 12. (Continued.)
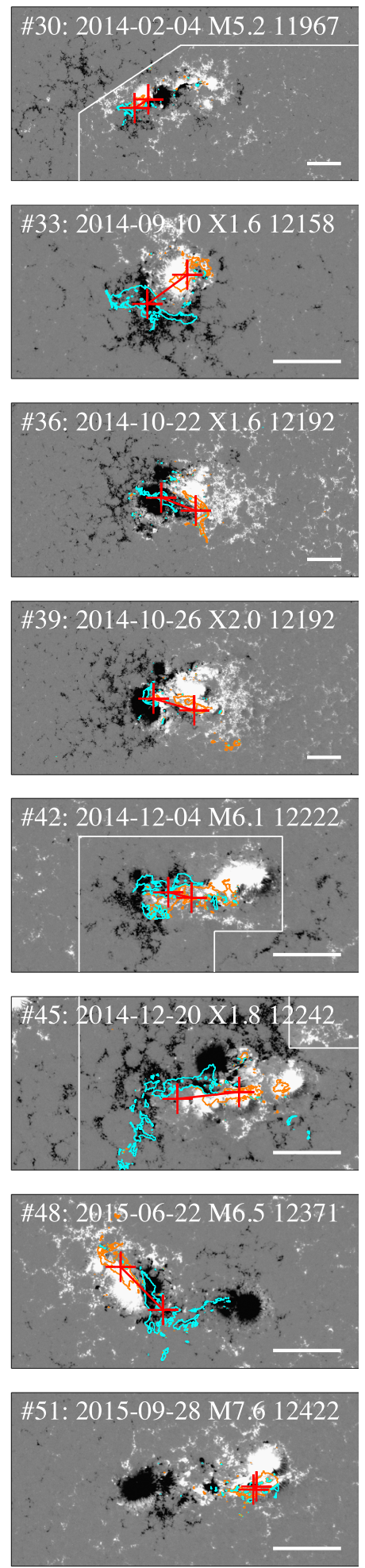

and

$$
\begin{aligned}
|\Phi|_{\mathrm{AR}, 2}= & {[2.3,3.4,2.1,5.2,4.0,4.4,1.9,7.6,8.5,14.0,} \\
& 15.8,16.6,16.6,15.8,14.2,2.9,3.4,5.3, \\
& 3.9] \times 10^{22} \mathrm{Mx} .
\end{aligned}
$$

We obtain $t=-3.229$ and $\nu=19.40\left(\nu^{*}=19\right)$. Since $t_{0.005}(19)=2.861$ and $t_{0.0005}(19)=3.883$, the probability is between 0.005 and 0.05 and the null hypothesis is rejected at $99.5 \%$ confidence, which is the same level as the test for the spot areas including AR 12192. 
Table 3

Measured Parameters of Flare Events

\begin{tabular}{|c|c|c|c|c|c|c|c|c|c|c|c|}
\hline \multirow{2}{*}{ Event \# } & \multicolumn{3}{|c|}{ GOES Parameters } & \multicolumn{3}{|c|}{ AR Parameters } & \multicolumn{4}{|c|}{ Flare Parameters } & \multirow{2}{*}{$\begin{array}{c}\mathrm{CME} \\
V_{\mathrm{CME}} \\
\left(\mathrm{km} \mathrm{s}^{-1}\right)\end{array}$} \\
\hline & $\begin{array}{l}\tau_{\text {FWHM }} \\
\text { (s) }\end{array}$ & $\begin{array}{c}\tau_{\text {decay }} \\
(\mathrm{s})\end{array}$ & $\left(10^{-4}{ }_{\mathrm{SXR}}^{\mathrm{W} \mathrm{m}^{-2}}\right)$ & $\begin{array}{c}S_{\text {spot }} \\
\text { (MSH) }\end{array}$ & $\begin{array}{c}|\Phi|_{\mathrm{AR}} \\
\left(10^{22} \mathrm{Mx}\right)\end{array}$ & $\begin{array}{c}\overline{|B|_{\mathrm{AR}}} \\
(\mathrm{G})\end{array}$ & $\begin{array}{l}S_{\text {ribbon }} \\
(\mathrm{MSH})\end{array}$ & $\begin{array}{l}d_{\text {ribbon }} \\
(\mathrm{Mm})\end{array}$ & $\begin{array}{c}|\Phi|_{\text {ribbon }} \\
\left(10^{21} \mathrm{Mx}\right)\end{array}$ & $\begin{array}{l}\mid \overline{|B|} \text { ribbon } \\
(\mathrm{G})\end{array}$ & \\
\hline 1 & 700 & 910 & 0.66 & 482 & 2.3 & 770 & 233 & 9.1 & 2.9 & 411 & $\ldots$ \\
\hline 2 & 772 & 429 & 2.2 & 678 & 2.8 & 685 & 380 & 21.1 & 5.7 & 496 & 669 \\
\hline 3 & 457 & 188 & 1.5 & 859 & 3.4 & 656 & 398 & 24.4 & 4.5 & 373 & $\ldots$ \\
\hline 4 & 219 & 71 & 0.93 & 303 & 2.1 & 662 & 170 & 16.8 & 1.4 & 270 & $\ldots$ \\
\hline 5 & 2249 & 522 & 0.60 & 391 & 2.8 & 666 & 465 & 29.8 & 4.2 & 301 & 610 \\
\hline 6 & 627 & 277 & 0.93 & 315 & 2.6 & 634 & 701 & 18.9 & 4.7 & 222 & 1315 \\
\hline 7 & 1176 & 986 & 0.53 & 288 & 2.9 & 753 & 102 & 13.6 & 1.1 & 360 & 782 \\
\hline 8 & 268 & 139 & 2.1 & 284 & 2.7 & 668 & 252 & 10.6 & 2.0 & 259 & 575 \\
\hline 9 & 399 & 130 & 1.8 & 220 & 2.5 & 625 & 126 & 18.7 & 1.2 & 314 & 792 \\
\hline 10 & 2748 & 918 & 0.87 & 379 & 2.8 & 589 & 678 & 41.1 & 6.3 & 305 & 2175 \\
\hline 11 & 1372 & 922 & 5.4 & 1256 & 3.8 & 588 & 1159 & 44.3 & 16.1 & 459 & 2684 \\
\hline 12 & 2699 & 1030 & 1.3 & 1241 & 3.8 & 601 & 539 & 53.1 & 8.3 & 509 & 1825 \\
\hline 13 & 2245 & 1004 & 0.63 & 960 & 3.8 & 614 & 661 & 54.0 & 7.2 & 359 & 950 \\
\hline 14 & 3883 & 1315 & 0.84 & 801 & 3.8 & 601 & 790 & 56.0 & 8.4 & 350 & 491 \\
\hline 15 & 387 & 183 & 0.57 & 1103 & 5.2 & 730 & 340 & 31.3 & 2.2 & 216 & $\ldots$ \\
\hline 16 & 506 & 103 & 0.56 & 682 & 3.2 & 703 & 428 & 8.1 & 2.2 & 170 & 313 \\
\hline 17 & 221 & 76 & 0.53 & 881 & 4.0 & 695 & 277 & 9.8 & 2.8 & 333 & $\ldots$ \\
\hline 18 & 311 & 85 & 0.61 & 1008 & 4.4 & 652 & 324 & 20.9 & 2.9 & 294 & $\ldots$ \\
\hline 19 & 3985 & 260 & 1.4 & 1369 & 6.8 & 693 & 562 & 42.5 & 9.2 & 541 & 885 \\
\hline 20 & 1198 & 362 & 0.65 & 218 & 1.5 & 795 & 654 & 17.4 & 2.8 & 143 & 861 \\
\hline 21 & 430 & 74 & 0.93 & 437 & 3.1 & 751 & 500 & 20.2 & 3.3 & 217 & 399 \\
\hline 22 & 373 & 111 & 0.63 & 308 & 2.4 & 752 & 300 & 16.3 & 2.9 & 316 & 268 \\
\hline 23 & 258 & 612 & 0.50 & 222 & 1.9 & 731 & 333 & 5.5 & 1.5 & 145 & $\ldots$ \\
\hline 24 & 164 & 57 & 3.3 & 979 & 4.9 & 594 & 191 & 4.1 & 1.5 & 254 & 562 \\
\hline 25 & 227 & 93 & 1.1 & 781 & 5.0 & 723 & 232 & 9.7 & 1.6 & 229 & 336 \\
\hline 26 & 330 & 105 & 1.1 & 602 & 4.5 & 704 & 262 & 4.7 & 1.6 & 207 & 413 \\
\hline 27 & 1624 & 433 & 0.64 & 433 & 3.0 & 668 & 431 & 23.4 & 5.2 & 395 & 271 \\
\hline 28 & 1526 & 460 & 0.72 & 1679 & 7.6 & 784 & 745 & 26.9 & 4.5 & 199 & $\ldots$ \\
\hline 29 & 2345 & 993 & 1.2 & 1617 & 7.9 & 796 & 863 & 41.7 & 3.3 & 127 & 1830 \\
\hline 30 & 440 & 148 & 0.52 & 1523 & 8.5 & 810 & 230 & 34.5 & 3.4 & 481 & $\ldots$ \\
\hline 31 & 444 & 212 & 1.0 & 126 & 1.1 & 666 & 150 & 5.9 & 1.4 & 301 & 528 \\
\hline 32 & 1606 & 428 & 0.73 & 347 & 2.8 & 674 & 680 & 29.8 & 4.4 & 215 & 1203 \\
\hline 33 & 2875 & 1986 & 1.6 & 492 & 3.3 & 745 & 666 & 52.7 & 8.2 & 407 & 1071 \\
\hline 34 & 1829 & 668 & 0.51 & 130 & 1.3 & 790 & 426 & 32.8 & 1.6 & 125 & 215 \\
\hline 35 & 2697 & 1028 & 0.87 & 2756 & 14.0 & 690 & 744 & 69.7 & 11.0 & 487 & $\ldots$ \\
\hline 36 & 2378 & 567 & 1.6 & 2877 & 15.8 & 655 & 656 & 79.1 & 9.5 & 479 & $\ldots$ \\
\hline 37 & 3404 & 968 & 3.1 & 2781 & 16.6 & 747 & 1639 & 74.4 & 15.3 & 308 & $\ldots$ \\
\hline 38 & 4790 & 1244 & 1.0 & 2786 & 16.6 & 744 & 790 & 105.1 & 11.2 & 466 & $\ldots$ \\
\hline 39 & 1937 & 805 & 2.0 & 2572 & 15.8 & 719 & 645 & 90.8 & 9.2 & 472 & $\ldots$ \\
\hline 40 & 1514 & 293 & 0.71 & 2293 & 14.2 & 693 & 634 & 27.7 & 6.7 & 347 & $\ldots$ \\
\hline 41 & 879 & 484 & 1.6 & 372 & 4.3 & 638 & 887 & 43.4 & 7.8 & 289 & 795 \\
\hline 42 & 2324 & 968 & 0.61 & 670 & 2.9 & 661 & 750 & 25.6 & 5.2 & 229 & $\ldots$ \\
\hline 43 & 2601 & 1022 & 0.87 & 899 & 4.5 & 680 & 252 & 49.6 & 4.5 & 590 & 587 \\
\hline 44 & 1876 & 812 & 0.69 & 921 & 3.4 & 705 & 407 & 39.0 & 5.0 & 402 & $\ldots$ \\
\hline 45 & 1976 & 1505 & 1.8 & 1267 & 5.3 & 568 & 806 & 66.3 & 9.4 & 385 & 830 \\
\hline 46 & 379 & 108 & 0.51 & 382 & 2.3 & 648 & 209 & 7.2 & 1.4 & 224 & 1040 \\
\hline 47 & 612 & 253 & 2.1 & 560 & 2.7 & 682 & 218 & 15.2 & 3.3 & 496 & 240 \\
\hline 48 & 3168 & 544 & 0.65 & 1120 & 4.6 & 699 & 648 & 64.6 & 8.1 & 412 & 1209 \\
\hline 49 & 3017 & 1886 & 0.79 & 697 & 3.8 & 635 & 1543 & 56.4 & 7.7 & 164 & 1627 \\
\hline 50 & 154 & 32 & 0.56 & 1264 & 5.3 & 706 & 113 & 7.0 & 0.9 & 256 & $\ldots$ \\
\hline 51 & 401 & 116 & 0.76 & 1026 & 3.9 & 639 & 271 & 4.6 & 2.2 & 266 & $\ldots$ \\
\hline $\max$ & 4790 & 1986 & 5.40 & 2877 & 16.6 & 810 & 1639 & 105.1 & 16.1 & 590 & 2684 \\
\hline $\min$ & 154 & 32 & 0.50 & 126 & 1.1 & 568 & 102 & 4.1 & 0.9 & 125 & 215 \\
\hline median & 1198 & 433 & 0.87 & 781 & 3.8 & 685 & 431 & 26.9 & 4.4 & 308 & 792 \\
\hline$\sigma$ & 1201 & 487 & 0.89 & 753 & 4.1 & 60 & 329 & 24.3 & 3.6 & 119 & 599 \\
\hline
\end{tabular}

Notes. The maximum, minimum, median, and standard deviation $(\sigma)$ of each parameter are shown at the bottom. The values for $V_{\mathrm{CME}}$ are calculated from the 32 eruptive events.

${ }^{a}$ Non-eruptive events are marked with ellipses. 


\section{REFERENCES}

Andrews, M. D. 2003, SoPh, 218, 261

Asai, A., Yokoyama, T., Shimojo, M., et al. 2004, ApJ, 611, 557

Aulanier, G., Démoulin, P., Schrijver, C. J., et al. 2013, A\&A, 549, A66

Benz, A. O., \& Güdel, M. 2010, ARA\&A, 48, 241

Bogart, R. S., Baldner, C., Basu, S., Haber, D. A., \& Rabello-Soares, M. C. 2011, JPhCS, 271, 012008

Bruzek, A. 1964, ApJ, 140, 746

Carmichael, H. 1964, NASSP, 50, 451

Carrington, R. C. 1859, MNRAS, 20, 13

Chatterjee, P., Hansteen, V., \& Carlsson, M. 2016, PhRvL, 116, 101101

Cheng, X., Zhang, J., Ding, M. D., Guo, Y., \& Su, J. T. 2011, ApJ, 732, 87

Cheung, M. C. M., \& Isobe, H. 2014, LRSP, 11, 3

Dodson, H. W. 1949, ApJ, 110, 382

Dodson, H. W., \& Hedeman, E. R. 1949, ApJ, 110, 242

Ellison, M. A. 1946, MNRAS, 106, 500

Ellison, M. A. 1949, MNRAS, 109, 3

Fan, Y. 2009, LRSP, 6, 4

Fan, Y., Zweibel, E. G., Linton, M. G., \& Fisher, G. H. 1998, ApJL, 505, L59

Fang, F., \& Fan, Y. 2015, ApJ, 806, 79

Forbush, S. E. 1946, PhRv, 70, 771

Gopalswamy, N., Lara, A., Kaiser, M. L., \& Bougeret, J.-L. 2001, JGR, 106, 25261

Harra, L., Schrijver, C. J., Janvier, M., et al. 2016, SoPh, 291, 1761

Hirayama, T. 1974, SoPh, 34, 323

Hodgson, R. 1859, MNRAS, 20, 15

Hudson, H. 2010, in Heliophysics: Space Storms and Radiation: Causes and Effects, ed. C. J. Schrijver \& G. L. Siscoe (Cambridge: Cambridge Univ. Press), 123

Kahler, S. W., Ling, A., \& White, S. M. 2015, SpWea, 13, 116

Khlystova, A. I., \& Sokoloff, D. D. 2009, ARep, 53, 281

Kleint, L., Battaglia, M., Reardon, K., et al. 2015, ApJ, 806, 9

Kliem, B., \& Török, T. 2006, PhRvL, 96, 255002

Kopp, R. A., \& Pneuman, G. W. 1976, SoPh, 50, 85

Künzel, H. 1960, AN, 285, 271

Kurokawa, H. 1989, SSRv, 51, 49

Lemen, J. R., Title, A. M., Akin, D. J., et al. 2012, SoPh, 275, 17

Linton, M. G., Longcope, D. W., \& Fisher, G. H. 1996, ApJ, 469, 954

Mackay, D. H., Karpen, J. T., Ballester, J. L., Schmieder, B., \& Aulanier, G. 2010, SSRv, 151, 333

Maehara, H., Shibayama, T., Notsu, S., et al. 2012, Natur, 485, 478
Maehara, H., Shibayama, T., Notsu, Y., et al. 2015, EP\&S, 67, 59

Möstl, C., Rollett, T., Frahm, R. A., et al. 2015, NatCo, 6, 7135

Mullan, D. J., Mathioudakis, M., Bloomfield, D. S., \& Christian, D. J. 2006, ApJS, 164, 173

Neher, H. V., \& Roesch, W. C. 1948, RvMP, 20, 350

Neupert, W. M. 1968, ApJL, 153, L59

Parker, E. N. 1955, ApJ, 121, 491

Pesnell, W. D., Thompson, B. J., \& Chamberlin, P. C. 2012, SoPh, 275, 3

Petschek, H. E. 1964, NASSP, 50, 425

Priest, E. R., \& Forbes, T. G. 2002, A\&ARv, 10, 313

Reale, F. 2007, A\&A, 471, 271

Richardson, R. S. 1948, ApJ, 107, 78

Sammis, I., Tang, F., \& Zirin, H. 2000, ApJ, 540, 583

Scherrer, P. H., Schou, J., Bush, R. I., et al. 2012, SoPh, 275, 207

Schou, J., Scherrer, P. H., Bush, R. I., et al. 2012, SoPh, 275, 229

Schrijver, C. J. 2007, ApJL, 655, L117

Schrijver, C. J., Aulanier, G., Title, A. M., Pariat, E., \& Delannée, C. 2011, ApJ, 738, 167

Schrijver, C. J., Beer, J., Baltensperger, U., Cliver, E. W., \& Güdel, M. 2012, JGRA, 117, A08103

Shibata, K., Isobe, H., Hillier, A., et al. 2013, PASJ, 65, 49

Shibata, K., \& Magara, T. 2011, LRSP, 8, 6

Shibata, K., \& Yokoyama, T. 1999, ApJL, 526, L49

Shibata, K., \& Yokoyama, T. 2002, ApJ, 577, 422

Sturrock, P. A. 1966, Natur, 211, 695

Sun, X., Bobra, M. G., Hoeksema, J. T., et al. 2015, ApJL, 804, L28

Takasao, S., Fan, Y., Cheung, M. C. M., \& Shibata, K. 2015, ApJ, 813, 112

Tanaka, K. 1991, SoPh, 136, 133

Thalmann, J. K., Su, Y., Temmer, M., \& Veronig, A. M. 2015, ApJL, 801 L23

Toriumi, S., Hayashi, K., \& Yokoyama, T. 2014a, ApJ, 794, 19

Toriumi, S., Iida, Y., Kusano, K., Bamba, Y., \& Imada, S. 2014b, SoPh, 289, 3351

Wang, R., Liu, Y. D., Yang, Z., \& Hu, H. 2014, ApJ, 791, 84

Wang, Y., \& Zhang, J. 2007, ApJ, 665, 1428

Wang, Y.-M., \& Sheeley, N. R., Jr. 1989, SoPh, 124, 81

Webb, D. F., \& Hundhausen, A. J. 1987, SoPh, 108, 383

Yashiro, S., Gopalswamy, N., Akiyama, S., Michalek, G., \& Howard, R. A. 2005, JGRA, 110, A12S05

Yokoyama, T., \& Shibata, K. 1997, ApJL, 474, L61

Yokoyama, T., \& Shibata, K. 1998, ApJL, 494, L113

Zirin, H., \& Liggett, M. A. 1987, SoPh, 113, 267 